$\underline{\text { Preprint typeset in JHEP style - HYPER VERSION }}$

hep-th/0602136

UFIFT-HEP-06-4

CU-TP-1144

\title{
Heating in Brane Inflation and Hidden Dark Matter
}

\author{
Xingang Chen ${ }^{1,2}$ and S.-H. Henry $\mathrm{Tye}^{3}$ \\ ${ }^{1}$ Institute for Fundamental Theory \\ Department of Physics, University of Florida, Gainesville, FL 32611 \\ ${ }^{2}$ ISCAP, Physics Department, Columbia University \\ New York, NY 10027 \\ ${ }^{3}$ Newman Laboratory for Elementary Particle Physics \\ Cornell University, Ithaca, NY 14853
}

\begin{abstract}
Towards the end of brane inflation, the brane pair annihilation produces massive closed strings. The transfer of this energy to Standard Model (SM) open string modes depends on where the SM branes and the brane annihilation are located: in the bulk, in the same throat or in different throats. We find that, in all cases as long as the brane annihilation and the SM branes are not both in the bulk, the transfer of energy to start the hot big bang epoch can be efficient enough to be compatible with big bang nucleosynthesis. The suppression of the abundance of the graviton and its Kaluza-Klein (KK) thermal relics follows from the warped geometry in flux compactification. This works out even in the scenarios where a long period of tunneling is expected. In the multi-throat scenario, we find a dynamical mechnism of selecting a long throat as the SM throat. We establish three new dark matter candidates: KK modes with specific angular momentum in the SM throat, those in the brane annihilation throat, and different matters generated by KK modes tunneled to other throats. Since the latter two couple to the visible matter sector only through graviton mediation, they behave as hidden dark matter. Hidden dark matter has novel implications on the dark matter coincidence problem and the high energy cosmic rays.
\end{abstract}




\section{Contents}

1. Introduction and summary 2

1.1 Introduction 2

1.2 Summary 6

2. KK modes in GKP compactification 9

2.1 KK modes in a throat 9

2.2 Tunneling between two throats

2.3 Resonance effect in tunneling 14

3. KK interactions

田. Production of KK modes from brane annihilation 21

5. Single throat heating 23

6. Double throat heating 26

6.1 KK evolution in A-throat 27

6.2 Tunneling from A-throat to S-throat 28

6.3 KK evolution in S-throat 30

7. The bulk case 32

8. Multi-throat heating 33

8.1 Throat deformation in Hubble expansion 33

8.2 Selection of long throats 34

8.3 Warped KK dark matter and hidden dark matter 35

9. Hidden dark matter 38

9.1 Hidden dark matter and dark matter coincidence problem 38

9.2 Hidden dark matter and cosmic rays 41

9.3 Comparison to other dark matters 43

10. Conclusions and discussions 44

丹. a KK cannot decay to all gravitons 46 


\section{Introduction and summary}

\subsection{Introduction}

One of the most interesting aspects of brane inflation [1, 2, 3] is its close connection to experiments. This includes the spectral index, tensor mode, non-Gaussianities and cosmic strings - ranging from astronomical observations, gravitational wave detections to cosmic microwave background radiation (CMBR) measurements.

Flux compactification in type IIB string theory has become an ideal setup to realize the brane inflation. This is not only because it provides promising mechanisms to stabilize moduli [4, [0, 6] and therefore a consistent string theory setup, but also because the flux induced warped space has become a very interesting new ingredient for model building.

First, warped throats provide different mechanisms to achieve brane inflation. AntiD3-branes naturally settle down in throats. The warped geometry red-shifts the brane tension and reduces (easily by a large factor) the mutual attraction between the branes and antibranes. This effect helps the slow-roll inflation, as in the KKLMMT scenario [7, [8]. Since the inflaton (the position of a D3-brane) is an open string mode, its kinetic term appears in the form of a DBI action. In warped space this imposes a speed-limit to slow down the brane motion in case the radiatively corrected potential becomes too steep. This mechanism generates the DBI inflation [9, 10, 11, 12]. Warped geometry together with the DBI action essentially guarantees enough e-folds of inflation. At the same time, effects of these two different mechanisms, and its combinations [13], can give rise to interesting observational signatures.

Second, the simple geometric picture allows us to explore variations of the simplest scenario involving multiple throats and branes. The multi-throat configuration arising from the flux compactification provides a natural setup to generate candidate inflatons [11]. Antibranes settle down in throats and annihilate against fluxes quantum mechanically [14, 15]. In 4-d view point, this process can start the brane inflationary epoch by eternally creating bubbles in the old inflation background. Within each bubble, the fluxantibrane annihilation naturally generate many D3-branes. The vacuum energy density within the bubble can still be large. These liberated D3-branes can roll out of the throat to other places and generate different types of brane inflation, such as the slow-roll or DBI inflation, whenever required conditions are satisfied. Different bubbles can also correspond to annihilations in different throats - the frequencies of which are determined by the relative lifetimes of the antibranes. In some cases, multiple branes may also be used to dynamically tune a flat potential [16].

Third, hierarchical range of string scales in multi-throat compactification provides different opportunities to observe stringy physics. Low string scale throat can make strings relatively easier to be observed in colliders [17], or can imprint stringy information on the density perturbations at large scale [12, 18]. High string scale throat can store 
high tension cosmic strings at the end of brane inflation. The allowed range of cosmic string tension overlaps with observational abilities 19, 20, 21, 22]. In this paper, we propose another possible consequence of brane inflation, namely hidden dark matter.

The crucial step that links the inflationary epoch to the hot big bang epoch is the heating at the end of the inflation. This is known as the graceful exit. However, the heating in both the single- and multi-throat configurations remain so far the least understood aspect of brane inflation. Namely, how can inflationary energy be efficiently transfered to heat up the Standard Model particles, and be compatible with the wellunderstood late-time cosmological evolution? This is the heating problem (also called reheating or preheating problem). To see why this is quite a non-trivial question, we first look at the end process of the brane inflation.

The brane inflation typically ends when branes annihilate antibranes. Significant insights have been gained into such a process recently [23, 24, 25]. Tachyonic modes appear when the brane-antibrane distance approaches the string scale and the annihilation process may be described by the tachyon rolling [26, 27]. No matter whether there are adjacent extra branes surviving such an annihilation, the initial end product is expected to be dominated by non-relativistic heavy closed strings [28, 29, 30]. These will further be involved to create lighter closed strings, KK modes, gravitons and open strings. (In this paper, we use KK modes as an abbreviation of the KK modes of massless closed strings.) We know from observations that, during big bang nucleosynthesis (BBN), the density of gravitons can be no more than a few percent of the total energy density of the universe. The rest is contributed by the Standard Model (SM) particles (mostly photons, neutrinos and electrons), which are open strings attached to a stack of SM (anti-)branes. We also know that the density of any non-relativistic relics can be no more than 10 times that of the baryons. Therefore the question becomes how the brane annihilation products, originally dominated by the closed string degrees of freedom, can eventually become the required light open string degrees of freedom living on the SM branes, with a negligible graviton density and a non-lethal amount of stable relics.

Depending on where the SM branes and brane annihilation are, we may consider 3 possibilities :

- when the SM branes are in the bulk (e.g., D7-branes wrapping a 4-cycle), while the brane annihilation happens in a throat; or vice versa; or they are both in the bulk;

- the single throat scenario, in which case the SM (anti-)branes are in the same throat where the inflationary brane annihilation takes place; note that the model can still have other throats around;

- the multi-throat scenario, where the inflationary branes annihilate in one throat (Athroat) while the SM branes are sitting in another throat (S-throat).

Both the slow-roll and DBI inflation can be realized in different scenarios. Depending on whether one uses the throat to solve the hierarchy problem or to stabilize the cosmic strings, different models have different requirements. For DBI inflation, a single A-throat 
may both fit the density perturbation and have a large hierarchy, because its prediction on the density perturbations can be independent of the A-throat warp factor [11, 12. In slow-roll brane inflationary models, the CMBR data typically requires only a modestly warped throat while solving the hierarchy problem requires substantially more warping, so the multi-throat scenario seems to be preferred as it can easily accommodate this very different warping properties. In both inflation models, the single throat versus the multi-throat scenario has another important implication. Brane annihilation is expected to produce all types of strings/defects that are not forbidden. This includes cosmic strings, which are simply massive large fundamental strings, D-1 strings and/or axionic strings. They tend to survive much better in a throat without branes. In the single throat scenario, due to their interaction with SM branes, they are unlikely to survive cosmologically due to their potential instabilities [21, 31, 32, 33]. So the prediction of cosmic strings is much firmer in the multi-throat scenario, provided heating after inflation is not a problem. This calls for a careful examination of heating in the multithroat scenario.

A number of studies have been done to address this heating problem 34, 35, 36, 37. An important observation is that, because the KK mode wave function is peaked at the bottom of the throat, its interaction with particles located at the IR side is much enhanced compared to that with the graviton. This is essentially along the line of Randall-Sundrum (RS) model [17]. Because of this, the graviton emission branching ratio during the brane decay and KK evolution is suppressed by powers of warp factors [34, 35, 36]. Such a suppression is absent if the brane annihilation happens in the bulk.

But several concerns have also been raised [34, 35, 36]. Since the KK modes in a throat can have (approximately) conserved angular momenta, it is important to estimate its relic density. This was previously estimated to be extremely large [35], and therefore any long-living KK relics seemed to be very dangerous to the BBN. Serious problems have also been raised in the multi-throat scenario where the question of heating become especially sharper [34, 36]. For example, in the multi-throat heating, the energy released from the brane annihilation has to be transfered to anther throat by tunneling, which is relatively a very long process. During this period, it seems that the KK modes in the Athroat is doing nothing but annihilate to gravitons. Requiring the initial annihilation rate to be smaller than the tunneling rate imposes very restrictive conditions on parameters of the model [36]. If generalized to the warped compactification where the tunneling rate is even much smaller, the analogous condition would rule out the multi-throat heating scenario.

However, a more detailed thermodynamic evolution of the heating process, especially that of the KK particles, has not been carefully studied. Such a thermal history is important for obtaining various estimates and assessing the viability of the brane inflation heating. This paper is a step towards this direction. Other new ingredients are, instead of the Randall-Sundrum (RS) 1-d warped geometry, we consider a 6-d warped geometry 
more relevant to the brane inflation in IIB theory (although the results can be easily evaluated and generalized to the RS case). This will cause interesting modifications in various estimates on tunneling rates and interaction rates. In a realistic compactification, throats are typically separated in the bulk, which tends to generate resonance effects in the tunneling from one throat to another. We expect the compactification volume to be dominated by the bulk, another important ingredient in the success of the graceful exit.

We will find many qualitatively different results compared to those in Ref. 34, 35, 36]. For example, the KK modes will decouple typically after they become non-relativistic. Because of the warping enhanced KK self-interactions, their relic density turn out to be suppressed by powers of warp factors and the bulk size. In the multi-throat case, the KK relic abundance is further suppressed due to an extra matter dominated tunneling phase. Also for the multi-throat case, although the graviton production rate is initially much greater than the tunneling rate, the rate gets diluted by the spatial expansion. So the graviton production is significant only in the early epoch and its eventual abundance is also suppressed by similar factors. The most stringent constraint on the tunneling is just that it cannot be too long to over-cool the universe.

So a final picture emerges is that a tower of KK particles gradually lowers as the universe expands, sometimes intervened by a long period of tunneling, and eventually transfers most of its entropy to the Standard Model particles at the bottom of the spectrum. The KK relic and graviton density are both reasonably suppressed by various factors due to the warped compactification.

During the course of this study, we will find many other interesting phenomena arising from the multi-throat heating. First, there is a dynamical process that selects a long throat to be heated. This is because the dense spectrum in long throats makes the level matching of the energy eigenstates, a necessary condition for tunneling between throats, easier to satisfy. This may provide a dynamical explanation of the selection of the RS type warp space as our Standard Model throat in the early universe. Second, we find several new dark matter candidates. For example, we propose a novel type of dark matter - the hidden dark matter - which is generated during the tunneling heating. The hidden dark matter has many unusual properties compared to the usual dark matter candidates.

Throughout the paper, we shall see the importance and richness of the warped space and multi-throat compactification appearing in many places, making them an even more attractive cosmological setup, in addition to the various aspects already mentioned at the beginning of this introduction.

To simplify the analysis, we ignore closed string modes other than the gravity sector. The lightest modes in the other relevant closed string sectors (such as moduli) may either be more massive and stabilized throughout the whole process; or become part of massive particle spectrum with similar properties, and their inclusion will not make a qualitative difference on the basic issues. Similar situations apply to the gravitino and 
its KK modes, so it is reasonable to expect that the gravitino density will not pose a serious problem cosmologically in this setup. In general, the qualitative properties of a simplified warp metric is very close to that of a more realistic metric, such as that of the Klebanov-Strassler (KS) throat [38], whose KK properties have been studied [39, 40]. The simplified metric of $S^{5}$ allows a simple analytic treatment so much of the physics is more transparent. In instances when the qualitative properties of the metric of the warped deformed conifold differ from that of $S^{5}$, we shall point that out explicitly.

\subsection{Summary}

To make reading easier, here we summarize by giving a brief history before the big bang nucleosynthesis according to the brane inflation heating in the multi-throat scenario. For simplicity, here we only keep the most important factors such as the warp factors $h_{A, S}$, the length scales of the throats $R_{A, S}$, the string scale $m_{s}$, the string couple $g_{s}$ and the Planck mass $M_{\mathrm{Pl}}$. The subscript $A$ stands for the A-throat where brane annihilation towards the end of inflation takes place, while the S-throat is where the SM branes sit.

Immediately after the inflation at $t_{0} \approx g_{s}^{1 / 2} h_{A}^{-2} M_{\mathrm{Pl}} / m_{s}^{2}$, all branes and antibranes annihilate in the A-throat and decay into heavy closed strings. The universe enters into a period of matter-dominated phase. These closed strings subsequently decay into gravitons and their KK modes. The branching ratio for graviton production is very small because the KK modes are exponentially peaked at the infrared tip of the A-throat, while the graviton, being the zero mode, is not localized. In fact, our estimate of the graviton production during this epoch is much smaller than that in Ref. [35] due to the bulk size suppression.

After a very short period $\Delta t \sim g_{s}^{-2} h_{A}^{-1} m_{s}^{-1}$, the decay ends and then quickly thermalize into a tower of relativistic KK particles at the bottom of the throat. The temperature is below the red-shifted string scale $h_{A} m_{s}$. The universe becomes radiation-dominated.

As the universe expands, the temperature drops and the tower of KK particles one by one become non-relativistic and then quickly fall out of equilibrium. Because of warped space enhanced KK self-interaction, the abundance of these frozen-out species are suppressed by the warp factor and bulk size.

This lasts until $t \sim h_{A}^{-2} R_{A}^{2} M_{\mathrm{Pl}}$ when the temperature drops below the lowest KK mode, $T \sim h_{A} R_{A}^{-1}$. The universe goes back to be matter-dominated.

These lowest KK modes, which stable against decay to gravitons, enter a long period of tunneling process to other throats, in some cases going through the bulk resonance. During this period, the KK particles are decoupled from each other because of the redshifting of the spatial expansion. The graviton production is negligible comparing to that in the early KK thermal history. Since the graviton density goes as $a^{-4}$ while the KK modes go like $a^{-3}$ ( $a$ is the scale factor), the graviton density rapidly becomes even more negligible. 
Tunneling between two throats requires the energy level matching, and the chance of matching becomes more frequent for longer throats, since longer throats have denser spectra. Also because of this, the KK particles tunneled into these throats quickly decay into lower KK levels and no longer tunnel back. Due to these reasons, long throats are preferentially heated during the tunneling. It is reasonable to assume that the Sthroat, at $\mathrm{TeV}$ scale, is most warped throat or among the most warped. So this process heats the S-throat, as well as generating possible new dark matter candidates in other throats. This long matter-dominated tunneling phase further suppresses the final KK relics abundance in the A-throat and the graviton abundance by delaying the Standard Model radiation-dominated phase.

By $t \sim \Gamma_{\text {tun }}^{-1}$, most energy has tunneled out of the A-throat. Here the tunneling rate $\Gamma_{\text {tun }}$ can be of order $h_{A}^{9} R_{A}^{-1}$ or $h_{A}^{17} R_{A}^{-1}$ depending on whether the bulk resonance happens or not. For example, in Ref. [7, 8, 13], matching brane inflation to the CMBR data, the warp factor $h_{A}$ may range roughly from $1 / 4$ to $10^{-3}$. This will yield a reasonable tunneling time for BBN. In this case, the most heated throat turns out to be the Sthroat. The KK particles in this throat thermalize to a much lower temperature and bring the universe back to be radiation-dominated. At this moment, the temperature in the S-throat can be above or below the local red-shifted string scale depending on the details.

A similar story then applies to the KK tower in the S-throat. The difference is that, after the temperature drops below the lowest KK mode at $t \sim h_{S}^{-2} R_{S}^{2} M_{\mathrm{Pl}}$, the universe remains radiation-dominated. This is because all the KK modes in the S-throat finally release most of their entropy to the Standard Model particles on the branes at the bottom of the mass spectrum. The KK relics is similarly suppressed as those in the A-throat by the bulk size and (much smaller) warp factors. This connects to the BBN.

The KK modes in the bulk will tunnel to the throats, mostly because, without warping, they are very massive, and their wavefunctions spread out throughout the bulk. So we do not expect any KK relics in the bulk for the resonance case. We can also easily replace the S-throat with a bulk SM brane and the similar story will apply after KK tunnels out of the A-throat.

Our calculations suggest that KK modes may supply three types of new dark matter candidates.

First, since the wave functions of KK modes with specific angular momentum in the S-throat have no overlap (or very suppressed overlap) with SM branes sitting at the bottom of the throat, they do not decay into SM particles. As a result, they can be stable enough to become dark matter. Note that these are gravitational KK modes and that the Standard Model particles do not have KK excitations. So they are like the warped KK modes [41, 42], but different from the KK dark matter suggested in Ref. [43, 44, 45]. They interact with the SM particles via the exchange of (unstable) s-wave KK modes. In this sense, they are not too different from other dark matter candidates, 
such as KK excitations of standard model particles or lightest supersymmetric particles, though they can have very different production and scattering cross-sections.

Second, KK modes in another long throat away from the SM branes can also be dark matter candidates. If there are branes in such a throat, the KK energy can be converted to open string modes, much like what happens in the S-throat. Since these dark matters interact with SM particles only via gravitons, they will be hidden dark matter. They can be detected via gravitational lensing and rotation curves, but not in collider experiments or underground dark matter searches. These dark mater can have novel implications on the dark matter coincidence problem.

Third, KK modes with specific angular momenta in the A-throat can also be quite stable. Their tunneling rate is highly suppressed compared to the s-wave KK modes that are responsible for tunneling in heating. This dark matter is formed as thermal relics much like the first type of the KK dark matter. But since they are decoupled from the SM particles except for graviton mediations, they also belong to hidden dark matter. Their masses are estimated to be very high, so their eventual tunneling to the Sthroat and decay/interaction can produce very energetic events. Since these high energy events originate within the range of our galaxy but from outside the SM branes, they can generate cosmic rays that violate the GZK bound.

This paper is organized as follows.

In Sec. 2, we study the wave functions of the KK modes in GKP type of string compactification. We also study the tunneling properties of the KK particles between different throats. We estimate the tunneling rates in different cases where the bulk resonance is present or absent.

In Sec. 3, we estimate the cross sections of various possible interactions in the same setup. In both Sec. 2 and 3, some quantitative differences between the GKP type and 5-d RS type setup are pointed out.

In Sec. 4 , we outline the end process of the brane inflation - the brane-antibrane annihilation - and its various products. This leads to the initial condition of our main study of the KK thermal history.

In Sec. 5, 6, 7 and 8, we study the thermal history of the KK particles in single throat, double throat, bulk and multi-throat cases, respectively. Estimates on the KK relic and graviton density are made. Interesting phenomena including the throat deformation in the Hubble background, the selection of long throats, the warped KK dark matter and the hidden dark matter are discussed.

In Sec. 9, we study more properties of the hidden dark matter, focusing on the dark matter coincidence problem and possible implications on cosmic rays.

Sec. 10 contains conclusions and discussions on future prospects. 


\section{KK modes in GKP compactification}

In this section, we study the wavefunction and dynamics of KK modes in throats. Previous studies on brane inflation heating have been focused on the geometry of the orbifolded RS-type [42, 34]. In this paper, we are mostly interested in the generalized GKP-type compactification [4, 5, 46, 38], namely, multiple throats with IR cutoffs whose UV sides are connected to a six-dimensional bulk. We will first study the wavefunction and spectrum of a single throat case, then the tunneling of KK modes between different throats.

\subsection{KK modes in a throat}

In the GKP compactification, a KS throat is induced by three-form RR and NSNS fluxes around a conifold singularity. Except for the deformation around the IR cutoff of the throat, the gravitational and RR background generated by fluxes are similar to that by D3-branes with the same D3-charge. Therefore the analyses of KK modes dynamics is similar to that in the absorption cross section of black holes which have been studied extensively in the past [47, 48, 49, 50, 51, 52, 53]. The difference here is that we will need to impose a different boundary condition, since we prepare our initial state inside a throat.

We take the metric of the throat to be

$$
d s^{2}=A^{-1 / 2}\left(-d t^{2}+d \mathbf{x}^{2}+h_{\mu \nu} d x^{\mu} d x^{\nu}\right)+A^{1 / 2}\left(d r^{2}+r^{2} d \Omega_{5}^{2}\right)
$$

where we consider a simplified warp metric

$$
A(r)=1+\frac{R^{4}}{r^{4}}
$$

The $r$ is the radial coordinate in six extra dimensions, and $R$ is the characteristic length scale of the warped space. The 4D KK modes arise as metric perturbations $h_{\mu \nu}$, whose s-wave we decompose as

$$
h_{\mu \nu}=\tilde{\psi}_{\mu \nu}(x) \phi(r) \text {. }
$$

The leading order equation of motion for the s-wave KK modes is a Laplacian equation in the background geometry,

$$
\frac{1}{\rho^{5}} \frac{d}{d \rho}\left(\rho^{5} \frac{d \phi}{d \rho}\right)+\left(1+\frac{(m R)^{4}}{\rho^{4}}\right) \phi=0,
$$

where $m^{2}=-p^{2}$ is the mass of the KK mode with $\tilde{\psi}_{\mu \nu} \propto e^{i p x}$, and we have defined $\rho \equiv m r$.

To solve this differential equation, we redefine

$$
\phi \equiv \rho^{-5 / 2} \tilde{\phi}
$$


and get

$$
\left[\frac{d^{2}}{d \rho^{2}}-\frac{15}{4 \rho^{2}}+1+\frac{(m R)^{4}}{\rho^{4}}\right] \tilde{\phi}=0 .
$$

This differential equation is of Schroedinger type with zero energy, and the effect of the curved background geometry on KK modes is a potential barrier. We will study the inner and outer regions of the barrier separately and match them.

The outer region is $\rho \gg m R$, where Eq. (2.6) is approximately

$$
\left[\frac{d^{2}}{d \rho^{2}}-\frac{15}{4 \rho^{2}}+1\right] \tilde{\phi}=0
$$

The solution is given by the Bessel functions

$$
\tilde{\phi}(\rho)=\rho^{1 / 2}\left(J_{2}(\rho)+i N_{2}(\rho)\right) .
$$

Here is the place where we have imposed the boundary condition, that is, we only consider outgoing wave in the large $\rho$ region, because this is the KK mode leaking out of the throat. We can expand the solution (2.8) in two different limits. For $\rho \gg 1$,

$$
\tilde{\phi}=\sqrt{\frac{2}{\pi}} e^{i\left(\rho-\frac{5 \pi}{4}\right)} .
$$

This describes an outgoing spherical wave. For $m R \ll \rho \ll 1$,

$$
\tilde{\phi}=-i \frac{4}{\pi \rho^{3 / 2}}+\cdots+\frac{1}{8} \rho^{5 / 2}+\cdots
$$

The first dots indicates the higher order imaginary terms that are related to the $i N_{2}(\rho)$ term in Eq. (2.8). The leading real term in Eq. (2.10) comes from the $J_{2}(\rho)$ term in Eq. (2.8).

The inner region is $\rho \ll m R$, where we can approximate Eq. (2.6) as

$$
\left[\frac{d^{2}}{d \rho^{2}}-\frac{15}{4 \rho^{2}}+\frac{(m R)^{4}}{\rho^{4}}\right] \tilde{\phi}=0 .
$$

Changing variables, one can also bring this equation to Bessel's differential equation and get

$$
\tilde{\phi}(\rho)=\frac{\rho^{1 / 2}}{m R}\left[A J_{2}\left(\frac{m^{2} R^{2}}{\rho}\right)+B N_{2}\left(\frac{m^{2} R^{2}}{\rho}\right)\right] .
$$

We can again expand this solution in two different limits. For $m^{2} R^{2} \ll \rho \ll m R$,

$$
\tilde{\phi}=A \frac{m^{3} R^{3}}{8 \rho^{3 / 2}}+\mathcal{O}\left(\frac{m^{7} R^{7}}{\rho^{7 / 2}}\right)-B \frac{4 \rho^{5 / 2}}{\pi m^{5} R^{5}}+\mathcal{O}\left(\frac{\rho^{1 / 2}}{m R}\right) .
$$


Matching (2.13) and (2.10) through $\rho \sim m R$, we can determine the leading orders of the coefficients $A$ and $B$ in Eq. (2.13) as

$$
\begin{aligned}
& A=-\frac{32 i}{\pi} m^{-3} R^{-3}, \\
& B=-\frac{\pi}{32} m^{5} R^{5} .
\end{aligned}
$$

Note that there is also an imaginary part in $B$. We ignore this contribution since our eventual goal is to calculate the leading tunneling probability, in which such an imaginary term only leads to higher order corrections. For $\rho \ll m^{2} R^{2}$,

$$
\tilde{\phi}=\sqrt{\frac{1}{2 \pi}} \frac{\rho}{m^{2} R^{2}}\left[(A-i B) e^{i\left(\frac{m^{2} R^{2}}{\rho}-\frac{5 \pi}{4}\right)}+(A+i B) e^{-i\left(\frac{m^{2} R^{2}}{\rho}-\frac{5 \pi}{4}\right)}\right] .
$$

This describes the outgoing and reflected incoming waves inside the effective potential barrier. To the leading order, they have the same amplitude. The small difference caused by the coefficient $B$ leads to the tunneling probability

$$
P=1-\left|\frac{A-i B}{A+i B}\right|^{2}=\frac{\pi^{2}}{2^{8}} m^{8} R^{8} .
$$

We can perform a simple check on this tunneling probability by comparing fluxes of the outgoing waves between the inner and outer regions. To do this, we list the leading behavior of the wave function $\phi$, defined in (2.5), up to an overall normalization,

$$
\begin{aligned}
\rho \ll m^{2} R^{2}: & \phi & =-i \frac{32 \sqrt{2}}{\pi^{3 / 2}} m^{-5} R^{-5} \rho^{-3 / 2} \cos \left(\frac{m^{2} R^{2}}{\rho}-\frac{5 \pi}{4}\right), \\
m^{2} R^{2} \ll \rho \ll 1: & \phi & =-\frac{4 i}{\pi} \rho^{-4}, \\
\rho \gg 1: & \phi & =\sqrt{\frac{2}{\pi}} \rho^{-5 / 2} e^{i\left(\rho-\frac{5 \pi}{4}\right)} .
\end{aligned}
$$

For $\rho \ll m^{2} R^{2}$ we have equal amount of outgoing and incoming wave, the flux of the outgoing component can be calculated by multiplying the flux density $\mathbf{j}=\frac{1}{2 i}\left(\phi_{\text {out }}^{*} \nabla \phi_{\text {out }}-\right.$ c.c. $)$ with the area of warped unit five-sphere, $\pi^{3} A(r)^{5 / 4} r^{5}$, and the warped unit longitudinal volume, $A(r)^{-1}$. Up to an overall normalization, we get

$$
\text { Flux }=\frac{\pi^{3}}{2 i}\left(\phi^{*} r^{5} \frac{\partial}{\partial r} \phi-\text { c.c. }\right)=512 m^{-12} R^{-8} .
$$

The region $m^{2} R^{2} \ll \rho \ll 1$ is the extension of the effective potential, and the KK particles are tunneling. For $\rho \gg 1$, we only have the tunneled outgoing KK particles. The corresponding flux is

$$
\text { Flux }=\frac{2 \pi^{2}}{m^{4}}
$$


Comparing Eq. (2.20) and (2.21), we get the tunneling probability (2.16). Here we have only considered the s-wave, the tunneling probability of the higher partial waves will be suppressed by factors of $m R$.

The mass quantization of the KK modes comes from the IR cutoff of the throat at $\rho_{0}=m r_{0}$. Such a boundary condition will translate into a constraint on the wavefunction (2.17) or its first derivative at $\rho_{0}$. The periodicity of the trigonometric function then leads to $\Delta\left(m^{2} R^{2} / \rho_{0}\right)=\pi$. So the KK mass is quantized in unit of

$$
\Delta m=\pi h_{0} R^{-1},
$$

where

$$
h_{0}=r_{0} / R
$$

is the IR warp factor. The more realistic KS throat has a more complicated smooth shape at the IR end, but we expect the qualitative behavior of the mass quantization is similar 40 .

\subsection{Tunneling between two throats}

KK modes in the bulk can also be absorbed by a throat. Such a process is well analyzed previously when people study the black hole absorption cross section 447, 18, 49, 50, 51, 52, 53]. Here the boundary condition is to require only incoming wave in the inner region. We list the leading behavior of the s-wave in three different regions,

$$
\begin{aligned}
\rho \gg 1: & \phi & =-i \frac{32 \sqrt{2}}{\pi^{3 / 2}} m^{-5} R^{-5} \rho^{-5 / 2} \cos \left(\rho-\frac{5 \pi}{4}\right), \\
m^{2} R^{2} \ll \rho \ll 1: & \phi & =-\frac{4 i}{\pi} m^{-5} R^{-5}, \\
\rho \ll m^{2} R^{2}: & \phi & =\sqrt{\frac{2}{\pi}} m^{-2} R^{-2} \rho^{-3 / 2} e^{i\left(\frac{m^{2} R^{2}}{\rho}-\frac{5 \pi}{4}\right)} .
\end{aligned}
$$

The tunneling probability turns out to be the same as Eq. (2.16). This leads to the absorption cross section for the s-wave [52, 53],

$$
\sigma=\frac{\pi^{4}}{8} m^{3} R^{8} .
$$

Now we imagine two throats - A and X. The A-throat is where brane and anti-brane annihilate and KK modes are generated. These KK modes can leak through the bulk and tunnel into another throat - X-throat, which is originally empty. We assume that the A and X-throat are separated by a distance $D$, and the bulk size is $L$. Let $h_{A}=r_{A} / R_{A}$ and $h_{X}=r_{X} / R_{X}$ be the respective IR warp factors. The tunneling between the two throats requires the matching of the mass levels in two throats, $n_{A} h_{A} R_{A}^{-1}=n_{X} h_{X} R_{X}^{-1}$, 
within the energy widths. Here $n_{A}$ and $n_{X}$ are the KK levels in the $\mathrm{A}$ and X-throat, respectively.

We will consider two interesting cases. In the first case, consider $L \gtrsim D \gtrsim m^{-1}$. The size of the bulk are large enough, so that the KK modes with mass $m$ may exist in the bulk. In this case, we assume such a mass quantization condition in the bulk is satisfied for $m$. The KK modes will go in two steps: first they tunnel out of the A-throat but remain in the bulk, propagating and bouncing around (in the extra dimensions), then gradually scatter and tunnel into the X-throat. For the first step, because of the tunneling probability (2.16) and the fact that the KK mode in A-throat has a bouncing period $2 h_{A}^{-1} R_{A}$, the tunneling rate is

$$
\Gamma_{A \rightarrow b u l k} \approx \frac{\pi^{2}}{16^{2}} n_{A}^{8} h_{A}^{9} R_{A}^{-1}
$$

For the second step, the absorption probability is given by the cross section

$$
P_{b u l k \rightarrow X} \approx \frac{\sigma}{L^{5}}
$$

and the bouncing period of KK modes in the extra dimensions is $L$. So the absorption rate is

$$
\Gamma_{b u l k \rightarrow X} \approx \frac{\sigma}{L^{6}} \approx \frac{\pi^{4}}{8} n_{A}^{3} h_{A}^{3} \frac{R_{X}^{8}}{R_{A}^{3} L^{6}} .
$$

The tunneling time for the KK particles to first go from the A-throat to the bulk and then from the bulk to the X-throat is the summation of these two tunneling time. So the final rate is the smaller of these two rates (2.28) and (2.30),

$$
\Gamma_{A \rightarrow X} \approx \operatorname{Min}\left(\Gamma_{A \rightarrow b u l k}, \Gamma_{\text {bulk } \rightarrow X}\right) .
$$

Because in (2.30), $L \gtrsim n_{A}^{-1} h_{A}^{-1} R_{A}$, we have $\Gamma_{b u l k \rightarrow X} \lesssim n_{A}^{9} h_{A}^{9} R_{X}^{8} / R_{A}^{9}$. So the tunneling rate for $n_{A} \sim 1, R_{X} \sim R_{A}$ is roughly

$$
\Gamma_{A \rightarrow X} \lesssim h_{A}^{9} R_{A}^{-1}
$$

The above analyses assumes that the energy of the KK modes in the A-throat satisfies the mass quantization condition of the bulk, namely the bulk KK mode also satisfies the mass matching condition as that for the two throats. This condition is the resonance effect that we will discuss in the next subsection.

The second case is that such a bulk mass quantization condition is not satisfied. This also includes the case where the bulk size is too small, $m<L^{-1}$. Assuming the $\mathrm{A}$ and X-throats are still well separated, $D \gg R_{A}, R_{X}$, the $\mathrm{KK}$ particle has to tunnel two barriers at one time, since they cannot propagate in the bulk. Approximately, the tunneling probability will be the product of the probability (2.16) for tunneling out and that for the absorption, and the rate for $n_{A} \sim 1$ will be

$$
\Gamma_{A \rightarrow X} \sim h_{A}^{17} R_{A}^{-1} .
$$


Of course one can always increase this tunneling rate by arranging special configuration of throats, for example bringing them closer and making the potential overlap. We will focus on the generic case.

\subsection{Resonance effect in tunneling}

There is an equivalent way of looking at the same problem involving the tunneling resonance effect in the presence of the bulk. Consider the same setup in the previous subsection. There are two barriers that the particle must tunnel through: the region between the A-throat and the bulk, $r_{1}<r<r_{2}$, and the region between the bulk and the X-throat, $r_{3}<r<r_{4}$. The KK modes have no barrier in the bulk, at $r_{2} \leq r \leq r_{3}$.

The tunneling rate, or transmission coefficient, is straightforward to obtain in the WKB approximation. Beginning with the first barrier, the A throat to the bulk, the relation between the coefficients of the incoming waves and the outgoing waves is given by (see Merzbacher [54])

$$
\begin{aligned}
& \frac{1}{2}\left(\begin{array}{cc}
\Theta_{A}+\frac{1}{\Theta_{A}} & i\left(\Theta_{A}-\frac{1}{\Theta_{A}}\right) \\
-i\left(\Theta_{A}-\frac{1}{\Theta_{A}}\right) & \Theta_{A}+\frac{1}{\Theta_{A}}
\end{array}\right), \\
& \Theta_{A}=2 \exp \left(\int_{\tau_{1}}^{\tau_{2}} d \tau \sqrt{V_{e f f}(\tau)}\right),
\end{aligned}
$$

where $\tau_{1}$ and $\tau_{2}$ are the classical turning point. Note that the effective energy in the analogous Schrödinger equation is zero. The variable $\tau$ and the effective potential is defined in Ref. [40]. The transmission coefficient from the A throat to the bulk is given by

$$
\begin{aligned}
P_{A \rightarrow \text { bulk }} & =4\left(\Theta_{A}+\frac{1}{\Theta_{A}}\right)^{-2} \\
& \simeq \frac{4}{\Theta_{A}^{2}}
\end{aligned}
$$

This WKB method amounts to solving the leading tunneling behavior of the differential equation (2.5) and (2.6). Indeed, evaluating (2.3.5), one obtains [4]

$$
P_{A \rightarrow \text { bulk }} \sim\left(n_{A} h_{A}\right)^{8}
$$

in agreement with the tunneling probability (2.16).

Next we consider the probability of tunneling from the A-throat to the X-throat via the bulk. Up to some pre-factors, the matrix relating the coefficients of the incoming wave from the A-throat to the outgoing wave in the $\mathrm{X}$-throat is given by

$$
\frac{1}{4}\left(\begin{array}{cc}
\Theta_{A}+\Theta_{A}^{-1} & i\left(\Theta_{A}-\Theta_{A}^{-1}\right) \\
-i\left(\Theta_{A}-\Theta_{A}^{-1}\right) & \Theta_{A}+\Theta_{A}^{-1}
\end{array}\right)\left(\begin{array}{cc}
e^{-i W} & 0 \\
0 & e^{i W}
\end{array}\right)\left(\begin{array}{cc}
\Theta_{X}+\Theta_{X}^{-1} & i\left(\Theta_{X}-\Theta_{X}^{-1}\right) \\
-i\left(\Theta_{X}-\Theta_{X}^{-1}\right) & \Theta_{X}+\Theta_{X}^{-1}
\end{array}\right),
$$


where $W$ is the integral over the bulk

$$
W=\int_{\tau_{2}}^{\tau_{3}} \sqrt{-V_{e f f}(\tau)} d \tau
$$

For simplicity we assume $R_{A}=R_{X}$. Even though the throats have different warp factors, the WKB integral over the barrier between the bulk and the X-throat ends up to be the same as that from the A-throat to the bulk, $\Theta_{X}=\Theta_{A}$, since the KK quantization mode $n_{A}$ and $n_{X}$ obey $n_{A} h_{A} R_{A}^{-1}=n_{X} h_{X} R_{X}^{-1}$.

So the transmission coefficient from the A-throat to the $\mathrm{X}$-throat via a bulk is given by

$$
P_{A \rightarrow X}=4\left(\left(\Theta_{A}^{2}+\frac{1}{\Theta_{A}^{2}}\right)^{2} \cos ^{2} W+4 \sin ^{2} W\right)^{-1} .
$$

In the absence of the bulk, $W=0$ and $T(A \rightarrow S)$ is very small,

$$
T_{A \rightarrow X} \sim \Theta_{A}^{-4} \sim\left(n_{A} h_{A}\right)^{16} .
$$

This implies a tunneling rate

$$
\Gamma_{A \rightarrow X} \sim n_{A}^{16} h_{A}^{17} R_{A}^{-1} .
$$

However, for KK modes satisfying

$$
W=\left(n_{W}+1 / 2\right) \pi
$$

so $\cos W=0$, the transmission coefficient approaches unity

$$
T_{A \rightarrow X} \sim 1
$$

This is the well-known resonance effect. Notice that the location of the transmission peaks is determined by the same quantum condition for the bound states in the bulk. For very massive KK modes or large bulk, one finds that the resonances easily occur. This means that tunneling from the A throat to another throat passing through the bulk may not be suppressed at all if the state is just at the resonance point. However, in any realistic model, the initial KK wave packet will have some widths which we have to average over. Suppose $h_{X} \ll h_{A}$. We expect a $n_{A}$ th KK mode will overlap with a small set of $n_{X}$ th KK modes so that the resonance effect will come into play. Or if some KK modes in the A-throat are in thermal equilibrium, then it is guaranteed that there are modes with the right energies to take advantage of this resonance effect. In the following we estimate the tunneling rate for these initial KK wave packets.

For large $\Theta_{A}$, so that the penetration through the barriers is strongly suppressed, the transmission coefficient $T$ has sharp narrow resonance peaks at these energies, with resonance width $\Delta \Gamma$. The distance between neighboring resonances is roughly

$$
D \simeq \frac{\pi}{\partial W / \partial E}
$$


Let us consider a wave packet that is localized in the A-throat at $t=0$. To study the behavior of the transmitted wave packet near a resonance, we take the initial wave packet to have a mean energy $E_{0}$ corresponding to a resonance. The packet has a width $\Delta E$ much bigger than the resonance width but much smaller than the distance between neighboring resonances, i.e.,

$$
D \gg \Delta E \gg \Delta \Gamma \text {. }
$$

Around the resonance,

$$
\cos W \simeq \mp\left(\frac{\partial W}{\partial E}\right)_{E=E_{0}}\left(E-E_{0}\right)
$$

and $\sin W \simeq 1$, so [54

$$
\sqrt{T} \simeq \mp \frac{\Delta \Gamma / 2}{E-E_{0}+i \Delta \Gamma / 2},
$$

where

$$
1 / \Delta \Gamma=\frac{\Theta_{A}^{2}}{4}\left(\frac{\partial W}{\partial E}\right)_{E=E_{0}} .
$$

For $\Delta E \gg \Delta \Gamma$, the total transmission probability for the incident wave packet is roughly $\Delta \Gamma / \Delta E$. One may interpret this result in the following way: the wave packet reaches the bulk in classical time. A fraction $\Delta \Gamma / \Delta E$ of the packet is transmitted to the X-throat according to the usual exponential decay law with a mean lifetime $1 / \Delta \Gamma$. The rest of the wave packet is reflected.

For the small band centered around the resonance point, using

$$
\frac{\partial W}{\partial E} \sim L \gtrsim h_{A}^{-1} R_{A},
$$

we get the tunneling rate

$$
\Gamma_{A \rightarrow X} \lesssim n_{A}^{9} h_{A}^{9} R_{A}^{-1},
$$

which agrees with (2.32). Note that this is effectively one barrier effect. This is because the resonance condition (2.43) is just the KK quantization condition in the bulk, so if the resonance happens, the wave packet can stay and bounce around in the bulk.

For a smooth distribution (e.g., a portion of a thermal distribution), the efficiency of transmission depends on $\Delta \Gamma, \Delta \Gamma / \Delta E$ and the time it takes to replenish the transmitted part of the wave packet. Assuming the thermalization time is much shorter than the tunneling time, the rate of transmission is

$$
\Gamma_{A \rightarrow X} \simeq \frac{\Delta \Gamma}{D} \Delta \Gamma \lesssim n_{A}^{17} h_{A}^{17} R_{A}^{-1},
$$


which agrees with that in Eq. (2.42) and (2.33). This can also be understood - a smooth distribution overall does not satisfy the resonance condition.

In summary, for an initial KK wave packet in the A-throat (e.g. with $n_{A}=1$ ), if the bulk resonance happens, the particles can propagate in the bulk and the tunneling rate is enhanced for these particles, $\Gamma_{A \rightarrow X} \sim h_{A}^{9} R_{A}^{-1}$. Otherwise, without resonance or for a smooth distribution, the rate is much smaller, $\Gamma_{A \rightarrow X} \sim h_{A}^{17} R_{A}^{-1}$. Notice that in either case, it is much smaller than $\Gamma \sim h^{5} R^{-1}$ for the RS 5-dimensional space-time case.

\section{KK interactions}

In this section, we study various cross sections of interactions among KK modes, gravitons and brane fields. Since we eventually only need an order-of-magnitude estimate of the thermal history, we will neglect the numerical factors for simplicity.

The interactions among the KK particles and gravitons are determined by the 10-dim action

$$
M_{10}^{8} \int d^{10} x \sqrt{-g_{10}} R_{10} \supset M_{10}^{8} \int d^{5} \Omega d r A r^{5} \int d^{4} x \sqrt{-g_{4}} R_{4} .
$$

We will first express various couplings in terms of the 10-d Planck mass $M_{10}$ and then convert it to 4 -d Planck mass $M_{\mathrm{Pl}}$. From the action (3.1), we can see that each throat contributes $M_{10}^{8} R_{i}^{6}$ to the 4-d Planck-mass-squared, while the bulk contributes $M_{10}^{8} L^{6}$. Since the typical situation in the multi-throat compactification is that the size of the bulk $L$ is bigger than the sum of length scales of all throats, $\sum R_{i}$, we approximate

$$
M_{\mathrm{Pl}} \approx M_{10}^{4} L^{3}
$$

Expanding (3.1) in terms of the 4-d metric fluctuations $h_{\mu \nu}$, we get

$$
M_{10}^{8} \int d^{5} \Omega d r A r^{5} \int d^{4} x(\partial h \cdot \partial h+h \cdot \partial h \cdot \partial h+h \cdot h \cdot \partial h \cdot \partial h+\cdots),
$$

where we only write the relevant expression schematically by neglecting indices that should be properly contracted with $\eta_{\mu \nu}$ and ignoring various numerical factors. After decomposing $h_{\mu \nu}=\sum \tilde{\psi}_{n \mu \nu}(x) \phi_{n}(\mathbf{r})$ as in (2.3) and integrating out the wave functions (2.17)-(2.19) in the extra dimensions, we can get couplings for the 4-d KK modes $\tilde{\psi}_{\mu \nu}$.

To make the kinetic term for the KK modes canonical, we first integrate the extra dimensions for the quadratic term. It turns out that the dominant contribution comes from the tip region $r \sim r_{0}$,

$$
M_{10}^{8} m^{-13} R^{-7} h_{0}^{-1} \int d^{4} x(\partial \tilde{\psi})^{2} .
$$

where $h_{0}$ is the warp factor (2.23). For the zero-mode graviton, the wave function in the extra dimension is a constant, for example, we will take it to be one. The quadratic 
term is dominated by the bulk

$$
M_{10}^{8} L^{6} \int d^{4} x\left(\partial \tilde{\psi}_{0}\right)^{2}
$$

Therefore the canonically normalized fields are

$$
\begin{aligned}
\psi & \equiv M_{10}^{4} m^{-13 / 2} R^{-7 / 2} h_{0}^{-1 / 2} \tilde{\psi} \\
\psi_{0} & \equiv M_{10}^{4} L^{3} \tilde{\psi}_{0} .
\end{aligned}
$$

We next look at the interactions. We consider the case where all KK particles and gravitons have similar energy. This applies to the case of the thermal equilibrium that we will focus on later. So in terms of orders of magnitude, the 3-point interaction $\lambda_{3} \int d^{4} x \psi \cdot \partial \psi \cdot \partial \psi$ corresponds to a cross section $\sigma \approx \lambda_{3}^{2}$, and the 4-point interaction $\lambda_{4} \int d^{4} x \psi \cdot \psi \cdot \partial \psi \cdot \partial \psi$ corresponds to a cross section $\sigma \approx \lambda_{4}^{2} E^{2}$, where $E$ is the energy of the particles. We first look at the cubic interactions.

Three KK modes: This is the KK self-interactions. Integrating along the throat in (3.3), the dominant contribution comes from the tip of the throat, where the KK modes wave function are peaked. In terms of the canonically normalized fields, we have

$$
M_{10}^{-4} R^{-3} h_{0}^{-1} \int d^{4} x \psi \cdot \partial \psi \cdot \partial \psi
$$

This corresponds to an interaction cross section

$$
\sigma \approx\left(\frac{L}{R}\right)^{6} \frac{h_{0}^{-2}}{M_{\mathrm{Pl}}^{2}} .
$$

Two KK modes and one graviton: This is the KK modes annihilation process. The coupling of this interaction is still dominated by the tip region $r \sim r_{0}$,

$$
M_{10}^{-4} L^{-3} \int d^{4} x \psi \cdot \partial \psi \cdot \partial \psi
$$

where one of $\psi$ is $\psi_{0}$. This leads to an annihilation cross section

$$
\sigma \approx \frac{1}{M_{\mathrm{Pl}}^{2}}
$$

One KK mode and two gravitons: Because of the KK momentum conservation, namely

$$
\int d r r^{5} A \phi(r)=0
$$

such a cross section vanishes. This equation is in fact the orthogonality condition between the KK and the zero mode, so this conclusion can be easily generalized to more arbitrary 
geometry or multi-gravitons. Since any compactification has a zero mode graviton with constant wavefunction [40], $n$-point function involving one KK mode and $n-1$ gravitons is proportional to the orthogonality condition between the $\mathrm{KK}$ and graviton mode. Hence the KK mode cannot decay to all gravitons directly. This conclusion still holds when all other terms in the action (3.1) are considered. The arguments are given in Appendix A. For higher KK mode, it can still decay to several lower KK modes plus graviton with a rate $\ll m^{3} / M_{\mathrm{Pl}}^{2}$. But for the lowest $\mathrm{KK}$ mode, the only way it can emit graviton is to annihilate or scatter another particle.

Three gravitons: The graviton self-interactions are dominated by the bulk, their coupling and cross section turns out to be the same as the KK annihilation case, $\sigma \approx$ $1 / M_{\mathrm{Pl}}^{2}$.

If branes exist at the IR tip of the throat, the open string fields on the brane will interact with KK particles and gravitons. These interactions are given by the following term in the action,

$$
\int d^{4} x d^{5} \Omega d r A^{1 / 2} \sqrt{-g} \frac{1}{2} G^{\mu \nu} \partial_{\mu} \tilde{H} \partial_{\nu} \tilde{H} \delta\left(r-r_{0}\right) \delta^{5}\left(\Omega-\Omega_{0}\right),
$$

where $G^{\mu \nu}=A^{1 / 2} g^{\mu \nu}, r_{0}$ and $\Omega_{0}$ are the radial and angular location of the D3-branes. The $\tilde{H}$ denotes the brane fields, which for example can be a Standard Model Higgs. Expanding (3.12) in terms of the 4-d fluctuations $h_{\mu \nu}$, one gets

$$
\frac{R^{2}}{2 r_{0}^{2}} \int d^{4} x\left(\eta^{\mu \nu} \partial_{\mu} \tilde{H} \partial_{\nu} \tilde{H}+h^{\mu \nu} \partial_{\mu} \tilde{H} \partial_{\nu} \tilde{H}+h \cdot h \cdot \partial \tilde{H} \cdot \partial \tilde{H}+\cdots\right)_{r=r_{0}}
$$

We denote the canonically normalized SM particle as

$$
H \equiv \frac{R}{r_{0}} \tilde{H}
$$

The presence of the D3-branes breaks the conservation of the KK momentum. So for example, a KK particle can hit the brane and break into two SM particles.

KK-SM interaction: Such a three-point interaction is determined by the second term in $(3.13)$,

$$
M_{10}^{-4} R^{-3} h_{0}^{-1} \int d^{4} x \tilde{\psi}^{\mu \nu} \partial_{\mu} H \partial_{\nu} H
$$

The cross section is

$$
\sigma \approx\left(\frac{L}{R}\right)^{6} \frac{h_{0}^{-2}}{M_{\mathrm{Pl}}^{2}}
$$

If the KK particle is replaced by a graviton, we get

$$
\sigma \approx \frac{1}{M_{\mathrm{Pl}}^{2}}
$$


The quartic interactions can be analyzed in a similar way. In the following, we summarize both the cubic and quartic interactions according to the interaction type. In all cases, the cubic interactions are the most important.

- KK self-interactions: This includes $K K+K K \rightarrow K K, K K+K K \rightarrow K K+K K$, $K K+K K \rightarrow K K+g$ and higher points interactions. The cross section of these three interactions are given by the three terms in

$$
\sigma_{K K} \approx\left(\frac{L}{R}\right)^{6} \frac{h_{0}^{-2}}{M_{\mathrm{Pl}}^{2}}\left(1+\frac{n_{E}^{2}}{N^{2}}+\frac{n_{E}^{2}}{N^{2}}\left(\frac{R}{L}\right)^{6} h_{0}^{2}+\cdots\right)
$$

respectively. Here $n_{E}$ parameterizes the particle energy scale, $E=n_{E} h_{0} R^{-1}$. For relativistic species, $n_{E}>n_{K K}$, and for non-relativistic species, $n_{E}=n_{K K}$. In the last two terms, the relations $R^{4} \approx N g_{s} / m_{s}^{4}$ and $M_{\mathrm{Pl}}^{2} \approx m_{s}^{8} L^{6} / g_{s}^{2}$ have been used. These two terms are negligible to the first, so we have

$$
\sigma_{K K} \approx\left(\frac{L}{R}\right)^{6} \frac{h_{0}^{-2}}{M_{\mathrm{Pl}}^{2}}
$$

Comparing to the RS case, we have the same warp factor enhancement $h_{0}^{-2}$. In addition, the cross section in the GKP case is further enhanced by the bulk size $L^{6} / R^{6}$.

- Hard graviton production from $K K$ annihilation: This includes $K K+K K \rightarrow g$, $K K+K K \rightarrow K K+g, K K+K K \rightarrow g+g$ and higher points interactions. The cross section for the first reaction is (3.10), and the third has an additional suppression factor $E^{2} / M_{\mathrm{Pl}}^{2}$. The second process does not entirely annihilate the KK modes and was included in the previous case as a KK self-interaction. But this reaction emits a hard graviton and can be interesting as we consider the graviton production. The cross section is $\frac{n_{E}^{2}}{N^{2}} \frac{1}{M_{\mathrm{Pl}}^{2}}$. So the first reaction dominates,

$$
\sigma_{K g} \approx \frac{1}{M_{\mathrm{Pl}}^{2}}
$$

- Graviton interactions: This includes $g+g \rightarrow g, g+g \rightarrow g+g, g+g \rightarrow H+H$, $g+g \rightarrow K K+K K$ and higher points interactions. The largest cross section is that of the first,

$$
\sigma_{g g} \approx \frac{1}{M_{\mathrm{Pl}}^{2}} .
$$

The latter three are suppressed by an additional factor of $E^{2} / M_{\mathrm{Pl}}^{2}$. So as usual the graviton interaction is Planck-mass suppressed. 
- KK-SM interactions: This includes $H+H \rightarrow K K, H+H \rightarrow K K+K K$ and higher points interactions. Their cross section is

$$
\begin{aligned}
\sigma_{H K} & \approx\left(\frac{L}{R}\right)^{6} \frac{h_{0}^{-2}}{M_{\mathrm{Pl}}^{2}}\left(1+\frac{n_{E}^{2}}{N^{2}}+\cdots\right) \\
& \approx\left(\frac{L}{R}\right)^{6} \frac{h_{0}^{-2}}{M_{\mathrm{Pl}}^{2}}
\end{aligned}
$$

- Hard graviton production from SM particles annihilation: This includes $H+H \rightarrow g$, $H+H \rightarrow g+K K, H+H \rightarrow g+g$ and higher points interactions. Their cross sections are

$$
\begin{aligned}
\sigma_{H g} & \approx \frac{1}{M_{\mathrm{Pl}}^{2}}\left(1+\frac{n_{E}^{2}}{N^{2}}+\frac{E^{2}}{M_{\mathrm{Pl}}^{2}}+\cdots\right) \\
& \approx \frac{1}{M_{\mathrm{Pl}}^{2}}
\end{aligned}
$$

We note that the wavefunction (2.17)-(2.19) used here is for s-wave. For higher partial wave, we will have a phase shift in the wave function and an extra angular part. The qualitative estimates we made in this section also apply to those KK modes with non-vanishing wavefunction at the bottom of the throat where the SM branes are sitting, such as those associated with $S^{3}$ in a realistic deformed warped conifold of the KS throat. There, the KK modes with $S^{2}$ angular momentum has vanishing wavefunctions at the origin, so they will interact with the SM particles via the exchange of the unstable s-wave KK modes.

\section{Production of KK modes from brane annihilation}

To study the heating process in brane inflation, we first look at the initial state from the brane-antibrane annihilation. We choose the time coordinate by choosing $\rho \approx M_{\mathrm{Pl}}^{2} / t^{2}$. So at the initial brane annihilation $\rho \approx h_{A}^{4} T_{3}$, we have $t_{0} \approx g_{s}^{1 / 2} h_{A}^{-2} M_{\mathrm{Pl}} / m_{s}^{2}$. The string dynamics in brane annihilation can be described by a boundary conformal field theory [23, 24, 25]. Calculations in the tree level [28] and loop level [29, 30] string amplitudes indicate that the dominant product is non-relativistic massive closed strings, no matter whether there are any branes left over from such an annihilation. These heavy closed strings will further decay to lighter states, such as lighter closed and open strings, gravitons and KK modes. The time scale for such annihilation and decay processes is of order red-shifted string scale, $\Delta t \sim g_{s}^{-2} h_{A}^{-1} m_{s}^{-1}$, up to a constant factor depending on string levels and configurations [55]. The $\Delta t$ is much smaller than $t_{0}$, so these decay happens right after $t_{0}$.

As we will check later (in Eq. (5.5)), it is easy to satisfy the condition that the spatial expansion of the universe is slow enough for these products to thermalize. So both the 
decay and thermalization time is smaller than $t_{0}$. Therefore the initial energy density of the thermalized gas is approximately the same as the initial brane tension $h_{A}^{4} T_{3}$. For a gas of relativistic particles with $g$ degrees of freedom, the thermalized temperature will be $T_{0} \approx\left(g g_{s}\right)^{-1 / 4} h_{A} m_{s}$. For $g>g_{s}^{-1}$ this is below the red-shifted string scale in the throat. Hence these thermalized particles should be the graviton KK modes if $T_{0}>h_{A} R_{A}^{-1}$, as well as the brane fields (zeroth open strings modes) if there are branes left.

Gravitons are also present as a decay product. Since their cross section is of order $M_{\mathrm{Pl}}^{-2}$, they are never in thermal equilibrium as long as time is larger than the Planck scale. So it is already important to estimate its initial abundance.

If the brane-antibrane annihilation are in the bulk, the graviton and the bulk KK modes couplings to the heavy closed strings are of the same order of magnitudes. Then the initial graviton density will be a sizable fraction of the total energy density. Since during the BBN, graviton density cannot exceed a few percent of the total energy density of the universe, a more detailed analyses is required to determine this fraction. The analyses in Ref. [36] indicates that this is problematic. (However see a solution at the end of Sec. 7.)

The situation is very different in the warped space. This is because, unlike the zeroth mode graviton, KK modes are peaked at the tip of the warped space. In the 5-dim RS setup, their coupling to the heavy strings located at the IR end of the warped space will be enhanced by a factor of $h_{A}^{-1}$ relative to the graviton. Hence the graviton production is suppressed by a factor of $h_{A}^{2}$ [34, 35, 36]. The case that we consider here in the GKP setup is essentially the same except for the quantitative details. From the analyses in Eqs. (3.15)-(3.17), we know that the suppression for the graviton production is greater - by a factor of

$$
\left(\frac{R}{L}\right)^{6} h_{A}^{2}
$$

To get an order of magnitude feeling, we note that, in fitting CMBR data, $R / L \sim 1 / 3$ to $1 / 100$ and $h_{A} \sim 1 / 4$ to $1 / 1000$ are reasonable for inflation in A-throat [7, 8, 13]. If inflation takes place as the D3-brane is coming out of another throat, as in the IR DBI case [11, 12], then the value of $h_{A}$ is more relaxed.

So in the presence of any reasonable warp factor, the dominant products after braneantibrane annihilation, string decay and particle thermalization is a tower of relativistic KK modes with possible brane fields at the bottom of the mass spectrum. ${ }^{1}$ The thermal history of such a gas will be the main focus of our next few sections. The study of the evolution of such states will eventually lead us to conditions at the time of BBN, and hence provides a test whether this is a viable heating.

\footnotetext{
${ }^{1}$ The initial KK can be absent if $T_{0}<h_{A} R_{A}^{-1}$, so we only have relativistic brane fields. This simplified case only happens for very small $N_{A}<g$ in the single throat case.
} 


\section{Single throat heating}

In this section, we study the time-dependent thermodynamics of KK modes in single throat case. In this case, the D3-anti-D3-brane annihilation, and therefore their eventual product KK particles, are in the same throat as the Standard Model branes. Our goal is to give an estimate of the relic KK mode and graviton production from the thermal history. To keep it more general, in this and next section, we will first obtain results in terms of general cross sections and tunneling rate, and then evaluate them using the results in GKP type compactification in Sec. 2 \& 3 . It can also be evaluated in the Randall-Sundrum setup.

Initially the Universe is dominated by a gas of relativistic KK modes that are created from brane and string decay. We know that the evolution of the energy density during such a period follows

$$
\rho=\frac{3 M_{\mathrm{Pl}}^{2}}{4 t^{2}} .
$$

If the KK particle self-interacting rate $\Gamma(t)$ is bigger than the Hubble expansion rate $H(t)$, the KK particles are in thermal equilibrium. We will check shortly that this is the case for the period that we are interested in. The density of the thermalized relativistic particles is determined by the temperature,

$$
\rho \approx \frac{\pi^{2}}{30} g T^{4},
$$

where $g$ is the number of degrees of freedom of all relativistic particles in equilibrium. These particles include the KK and SM particles and their possible super-partners. For simplicity, we will take $g$ to be a constant of the same order of magnitude as the SM particles $\sim 100$. Variation of $g$ may come if the KK tower is very high at the early epoch, or in the absence of the SM particles. We will encounter such cases in the next section. The difference caused by the variation in $g$ is not essential in this section.

The time dependence of temperature $T$ then follows from Eq. (5.1) and (5.2),

$$
T(t) \approx g^{-1 / 4}\left(\frac{M_{\mathrm{Pl}}}{t}\right)^{1 / 2} .
$$

As the universe expands, the temperature decreases. Each KK mode consecutively becomes non-relativistic as $T$ drops below its mass.

At $T_{n r} \approx m=n_{K K} h_{0} R^{-1}$, the temperature crosses the mass of the $n_{K K}$ th KK modes (including all possible degenerate states) and these KK particles are becoming non-relativistic. From (5.3), the corresponding time is

$$
t_{n r} \approx g^{-1 / 2} M_{\mathrm{Pl}} m^{-2} .
$$


The condition that we can use the thermodynamics for this KK mode until this moment is

$$
\Gamma / H \approx \frac{n \sigma_{K K} v}{1 /\left(2 t_{n r}\right)} \approx g T^{3} \sigma_{K K} t_{n r} \gtrsim 1
$$

where $n$ is the number density of this KK mode and the velocity $v$ of the KK particle is approximated as 1 . This is easy to satisfy. For example, for $\sigma_{K K}$ in (3.19),

$$
\Gamma / H \approx g^{1 / 2} n_{K K} R^{-1} M_{\mathrm{Pl}}^{-1}\left(\frac{L}{R}\right)^{6} h_{0}^{-1} .
$$

If $g^{1 / 2} \approx 10, n_{K K} \approx 1, m_{s} \approx 10^{10} \mathrm{GeV}, N_{A}^{1 / 4} \approx 10\left(R_{A}^{4} \approx g_{s} N_{A} m_{s}^{-4}\right)$, and $M_{\mathrm{Pl}} \approx$ $10^{18} \mathrm{GeV}$, we need $\left(\frac{L}{R}\right)^{6} h_{0}^{-1}>10^{8}$. This can be easily satisfied e.g. by $L \gtrsim 10 R$ and $h_{0} \lesssim 0.01$.

The density of these non-relativistic KK particle will drop exponentially with temperature

$$
n_{n r}=g\left(\frac{m T}{2 \pi}\right)^{3 / 2} e^{-m / T}
$$

and their entropy is released to the other relativistic species. The self-interacting rate of such a particle also drops accordingly and this species quickly becomes decoupled. After $t_{n r}$, the decrease of the interaction rate $\Gamma=n_{n r} \sigma_{K K} v$ is dominated by the exponential decrease of $n_{n r}$. So when the KK particle is decoupled, $\Gamma \approx H\left(t_{d e c}\right)$, we can estimate the leading order of the particle number density as

$$
n_{\text {dec }}^{K K} \approx H\left(t_{d e c}\right) \sigma_{K K}^{-1} v^{-1}\left(t_{d e c}\right) \approx H\left(t_{n r}\right) \sigma_{K K}^{-1} \approx g^{1 / 2} M_{\mathrm{Pl}}^{-1} m^{2} \sigma_{K K}^{-1} .
$$

Here the changes in $H$ and $v$ from $t_{n r}$ to $t_{d e c}$ are neglected for simplicity. ${ }^{2}$ These changes will give further suppression factor which is logarithmically weaker.

The whole tower of KK modes is gradually lowered in this fashion, until the temperature drops below the mass of the last $\mathrm{KK}$ mode $h_{0} R^{-1}$. At this point, all $\mathrm{KK}$ particles are non-relativistic and release most of their entropy to the relativistic SM particles.

If any KK modes are stable because they carry some conserved quantity, they become relics after decoupling. It is important to estimate their relative abundance to the other relativistic SM particles. Since non-relativistic KK particles red-shift differently from relativistic SM particles, this relative abundance will be amplified in later evolution.

\footnotetext{
${ }^{2}$ Using (5.7), we can estimate $T\left(t_{d e c}\right) \approx m\left(\ln \frac{g m^{3}}{n_{d e c}}\right)^{-1}$. So, the velocity $v$ at $t_{d e c}$ is $v \approx \sqrt{T / m} \approx$ $\left(\ln \frac{g m^{3}}{n_{d e c}}\right)^{-1 / 2}$, which changes logarithmically slower than $n_{K K}$, consistent with our assumption. The same happens to $H$. An overall correction is a factor of $\left(\ln \frac{g m^{3}}{n_{\text {dec }}^{K K}}\right)^{-3 / 2}$, which is smaller than one and makes the $n_{\text {dec }}^{K K}$ in $(5.8)$ smaller.
} 
This amplification lasts until the transition epoch from radiation domination to matter domination (RDMD), at which time the density of these non-relativistic relics cannot be more than that of the radiations, even if they account for all the dark matter.

We now estimate this relative ratio. At $t_{d e c}$, the ratio of the energy density of the decoupled KK particle to the radiation energy density is

$$
\left(\frac{\rho^{K K}}{\rho^{t o t}}\right)_{t_{d e c}} \approx \frac{n_{\text {dec }} m}{g m^{4}} \approx \frac{1}{g^{1 / 2} m M_{\mathrm{Pl}} \sigma_{K K}} .
$$

Summing over all possible stable KK modes and taking into account of the red-shift, at the time of the radiation-matter transition, we have

$$
\begin{aligned}
\Omega_{R D M D}^{K K} & \equiv\left(\frac{\rho^{K K}}{\rho^{t o t}}\right)_{R D M D} \\
& =\sum_{n_{K K}}\left(\frac{\rho^{K K}}{\rho^{t o t}}\right)_{t_{d e c}} \frac{a\left(t_{R D M D}\right)}{a\left(t_{d e c}\right)} \\
& =\sum_{n_{K K}}\left(\frac{\rho^{K K}}{\rho^{t o t}}\right)_{t_{d e c}}\left(\frac{\rho^{t o t}\left(t_{d e c}\right)}{\rho_{R D M D}}\right)^{1 / 4} \\
& \approx \sum_{n_{K} K} g^{-1 / 4} M_{\mathrm{Pl}}^{-1} \rho_{R D M D}^{-1 / 4} \sigma_{K K}^{-1}
\end{aligned}
$$

where $\rho_{R D M D}$ is the energy density at the time of RDMD transition. Using the cross section $\sigma_{K K}$ in (3.19), we have ${ }^{3}$

$$
\Omega_{R D M D}^{K K} \approx \sum_{n_{K K}} g^{-1 / 4}\left(\frac{R}{L}\right)^{6} \frac{M_{\mathrm{Pl}}}{\rho_{R D M D}^{1 / 4}} h_{0}^{2} .
$$

For the single throat case, this ratio can be made very small if $h_{0}$ is small. Since $\rho_{R D M D}^{1 / 4} \approx 1 \mathrm{eV}, M_{\mathrm{Pl}} \approx 10^{18} \mathrm{GeV}$, we need $\left(\frac{R}{L}\right)^{6} h_{0}^{2} \lesssim 10^{-27}$. For example, this can be satisfied if $L \approx 100 R$ and $h_{0} \approx 10^{-8}$. A few comments are in order here. First, we can also evaluate (5.10) in the 5-d RS case. It is still suppressed by the warp factor $h_{0}^{2}$ but without the bulk size suppression. So the condition becomes more restrictive, $h_{0} \lesssim 10^{-14}$. Second, as we will see in the next section, $\Omega_{R D M D}^{K K}$ will receive another suppression factor in the double throat case due to an extra matter-dominated phase. Third, we note that we have been interested in the relic density resulted from the thermal history. Whether these relics can be stable enough is also important and case dependent [35, 15]. In this paper, we show that even the most restrictive case, namely the stable relics, is compatible with a successful heating.

\footnotetext{
${ }^{3}$ The cross section of the KK self-interaction can be enhanced by the KK-SM particles interactions (3.22). Especially when we have many different SM particles. Such an enhancement can further decrease the ratio.
} 
We now estimate the graviton production. They are produced through the KK and SM particles annihilation. Once produced, they are always decoupled and red-shifted as radiation. Therefore their density is

$$
\rho^{g r a v}(t) \approx \int_{t_{0}}^{t} T\left(t_{1}\right) n^{2}\left(t_{1}\right) \sigma_{K g} \frac{a^{4}\left(t_{1}\right)}{a^{4}(t)} d t_{1},
$$

where we estimate the graviton energy as $T$, which is the energy scale of the reactions involved. The $n \approx g T^{3}$ is the number density of the KK or SM particles. From (3.20) and (3.23) we know that $\sigma_{H g} \approx \sigma_{K g}$. The initial time $t_{0}$ is set by initial energy density released from the brane annihilation, $H_{0}^{2} \approx \frac{1}{4 t_{0}^{2}} \approx \frac{T_{3} h_{0}^{4}}{3 M_{\mathrm{Pl}}^{2}}$, as in Sec. . So we get

$$
\rho^{g r a v}(t) \approx g_{s}^{-1 / 4} g^{1 / 4} M_{\mathrm{Pl}}^{3} m_{s} \sigma_{K g} h_{0} \frac{1}{t^{2}} .
$$

In this integration, we can see that the dominant contribution comes from the initial moment $t_{0}$ over a period of order $t_{0}$. After that, $\rho^{\text {grav }}$ just red-shifts as $1 / t^{2}$. Therefore the faction of gravitons in total radiation is

$$
\Omega_{B B N \text { or } R D M D}^{\text {grav }} \equiv\left(\frac{\rho^{g r a v}}{\rho^{\text {tot }}}\right)_{B B N \text { or } R D M D} \approx g_{s}^{-1 / 4} g^{1 / 4} M_{\mathrm{Pl}} m_{s} \sigma_{K g} h_{0} .
$$

Using the cross section (3.20), we have

$$
\Omega_{B B N \text { or } R D M D}^{\text {grav }} \approx g_{s}^{-1 / 4} g^{1 / 4} \frac{m_{s}}{M_{\mathrm{Pl}}} h_{0},
$$

which is naturally very small. ${ }^{4}$ We note that the ratio (5.14) is proportional to the graviton production cross section $\sigma_{K g}$, but is independent of the KK self-interactions cross section $\sigma_{K K}$. This is the difference between the thermal dynamics and simple reactions. That is, KK and SM particles are in thermal equilibrium and their density is determined by the temperature rather than the cross section, while the gravitons are never in thermal equilibrium. The total graviton abundance is the summation of (4.1) and (5.15).

\section{Double throat heating}

In this section, we consider the heating process in double throat case. In this case, the brane-anti-brane annihilation happens in the A-throat and there is no brane left in the end. The Standard Model lives on the branes in the S-throat. So successful heating requires efficient energy transfer from KK modes in the A-throat to branes in the S-throat. We study this process in three steps: the evolution of KK modes in the A-throat, the tunneling between A and S-throat and the evolution of KK modes in the S-throat.

\footnotetext{
${ }^{4}$ Higher KK particle can also decay to several lower KK particles plus graviton with a rate $\ll m^{3} / M_{\mathrm{Pl}}^{2}$. Within a time interval $t_{n r} \approx g^{-1 / 2} M_{\mathrm{Pl}} m^{-2}$, the decayed fraction is $\ll g_{s}^{-1 / 4} g^{-1 / 2} N^{-1 / 4} \frac{m_{s}}{M_{\mathrm{Pl}}} h$, much smaller than (5.15).
} 


\subsection{KK evolution in A-throat}

The thermal history of the KK modes in the A-throat is very similar to that in the single throat case. The main difference is that at the bottom of the spectrum there is no longer SM particles. So even if the KK tower starts not very high, the particle degrees of freedom will not remain constant. We write the relation between $g$ and the temperature $T$ in the following ansatz

$$
g \approx n_{K K}^{\gamma} \approx\left(\frac{T}{h_{A} R_{A}^{-1}}\right)^{\gamma} \approx \tilde{\alpha}^{1+\frac{\gamma}{4}}\left(\frac{T}{h_{A} T_{3}^{1 / 4}}\right)^{\gamma},
$$

where the value of $\gamma$ depends on the degeneracy of the states. For example, for 5-d RS case, $\gamma=1$; for $A d S_{5} \times S^{5}, \gamma \approx 5$; for $A d S_{5} \times T^{11}, \gamma \approx 4$ to 5. Sometimes, as in the last

step, we will also write $R$ in terms of brane tension $T_{3}$, where $\tilde{\alpha} \approx N^{\frac{\gamma}{\gamma+4}}$. Therefore the thermal density of the KK modes is

$$
\rho(t) \approx \frac{T^{\gamma+4}}{\left(h_{A} R_{A}^{-1}\right)^{\gamma}},
$$

and the time dependence of the temperature follows

$$
T(t) \approx\left(h_{A} R_{A}^{-1}\right)^{\frac{\gamma}{\gamma+4}}\left(\frac{M_{\mathrm{Pl}}}{t}\right)^{\frac{2}{\gamma+4}} .
$$

The KK modes at each level $n_{K K}$ become non-relativistic when the temperature drops below $m \approx n_{K K} h_{A} R_{A}^{-1}$ at

$$
\begin{aligned}
t_{\text {Anr }} & \approx\left(h_{A} R_{A}^{-1}\right)^{\frac{\gamma}{2}} M_{\mathrm{Pl}} m^{-\frac{\gamma+4}{2}} \approx n_{K K}^{-\frac{\gamma+4}{2}} R_{A}^{2} M_{\mathrm{Pl}} h_{A}^{-2} \\
& \approx t_{\text {Adec }} .
\end{aligned}
$$

The decoupling time $t_{\text {Adec }}$ for these KK modes follows shortly after $t_{\text {Anr }}$. The main difference between this case and the previous single throat case is that, as the temperature drops below the last KK mode in the A-throat, the universe enters a period of matter dominated (MD) phase. The lowest KK mode stays in the A-throat waiting to tunnel. The tunneling time $t_{t u n} \approx \Gamma_{\text {tun }}^{-1}$ is estimated in Sec. 目.

The higher partial waves of KK modes take much longer time to tunnel, and if stable enough, can become non-relativistic relics. This relics density can be estimated at the time when they become decoupled. The corresponding number density is

$$
n_{\text {Adec }} \approx \frac{H\left(t_{A n r}\right)}{\sigma_{K K}} \approx t_{A n r}^{-1} \sigma_{K K}^{-1} .
$$

The relative density of these KK relics at decoupling is

$$
\left(\frac{\rho^{K K}}{\rho^{\text {tot }}}\right)_{t_{\text {Adec }}} \approx \frac{n_{\text {Adec }} m}{M_{\mathrm{Pl}}^{2} / t_{\text {Adec }}^{2}} .
$$


This ratio will be amplified during any radiation domination epoch that follows. As we will see, this will start from when the tunneling is done $\left(\approx t_{\text {tun }}\right)$ and lasts until the RDMD transition in the S-throat. So at RDMD, the relics abundance is

$$
\begin{aligned}
\Omega_{R D M D}^{K K} \equiv\left(\frac{\rho^{K K}}{\rho^{t o t}}\right)_{R D M D} & \approx \sum_{n_{K K}}\left(\frac{\rho^{K K}}{\rho^{\text {tot }}}\right)_{t_{\text {Adec }}} \frac{M_{\mathrm{Pl}}^{1 / 2} t_{\text {tun }}^{-1 / 2}}{\rho_{R D M D}^{1 / 4}} \\
& \approx \sum_{n_{K K}} n_{K K}^{-\gamma / 4} M_{\mathrm{Pl}}^{-1} \rho_{R D M D}^{-1 / 4} \sigma_{K K}^{-1}\left(\frac{t_{A n r}}{t_{\text {tun }}}\right)^{1 / 2} .
\end{aligned}
$$

The main difference between Eq. (6.7) and (5.10) is the last factor in Eq. (6.7). This further suppression is due to the matter dominant phase from $t_{A n r}$ to $t_{t u n}$. In the single throat case, after the last KK mode becomes non-relativistic at $t_{n r}$, the universe remains radiation dominated. In the double throat case, radiation dominance is delayed until $t_{\text {tun }}$.

The graviton production during this period can be estimated using Eq. (5.12). Similarly the integration is mainly contributed from the early epoch around $t_{0}$, where $t_{0}$ is determined by the initial condition $H_{0}^{2} \approx \frac{1}{4 t_{0}^{2}} \approx \frac{T_{3} h_{A}^{4}}{3 M_{\mathrm{P} 1}^{2}}$. We get

$$
\rho_{A}^{\text {grav }} \approx g_{s}^{-1 / 4} \tilde{\alpha}^{1 / 4} h_{A} m_{s} M_{\mathrm{Pl}}^{3} \sigma_{K g} \frac{1}{t^{2}} .
$$

The relative graviton density is

$$
\Omega_{B B N \text { or } R D M D}^{\text {grav }} \equiv\left(\frac{\rho_{A}^{\text {grav }}}{\rho_{A}^{\text {tot }}}\right)_{B B N \text { or } R D M D} \approx g_{s}^{-1 / 4} \tilde{\alpha}^{1 / 4} m_{s} M_{\mathrm{Pl}} \sigma_{K g} h_{A}\left(\frac{t_{A n r}}{t_{\text {tun }}}\right)^{2 / 3} .
$$

The last dilution factor is again due to the additional matter dominated phase from $t_{A n r}$ to $t_{t u n}$. Using (3.20) we see that this is naturally a very small ratio.

\subsection{Tunneling from A-throat to S-throat}

Because of the very small tunneling rate (2.31) and (2.32), the tunneling of the KK modes in A-throat effectively happens well after the temperature drops below the mass of the lowest KK mode. Therefore the tunneling is dominated by these non-relativistic lowest KK particles. The only way for the lowest KK mode to emit graviton is through interaction with other particles. But during the tunneling, they actually have long been decoupled from each other and their kinetic energy has been largely red-shifted. (For this gas, we know that one can assign an effective temperature $T \propto a^{-2} \propto t^{-4 / 3}$ if we keep a correspondingly changing chemical potential.) The energy density evolves similarly as in Eq. (5.1) except for a different numerical factor $4 / 3$. To get the total graviton production, we integrate all particles as in Eq. (5.12). Although the tunneling time scale tends to be very long as we see from Sec. 2, the graviton production from the KK annihilation in this period is negligible comparing to (6.9), as we can see from a similar 
integration as in Eq. (5.12). This is because the spatial expansion keeps on red-shifting the KK density and velocity.

So the only constraint on the time scale of this period is that the spatial expansion during this long period does not cool the universe too much. This requires that the energy density at the end of the tunneling, $\rho_{K K}\left(t_{t u n}\right) \approx M_{\mathrm{Pl}}^{2} \Gamma_{\text {tun }}^{2}$, be larger than the energy density during BBN, $(1 \mathrm{MeV})^{4}$. This gives a lower bound on $h_{A}$. For example, for the resonance case or the non-resonance case,

$$
\Gamma_{\text {tun }} \sim h_{A}^{9} R_{A}^{-1} \quad \text { or } \quad \Gamma_{\text {tun }} \sim h_{A}^{17} R_{A}^{-1}
$$

we need

$$
h_{A} \gtrsim\left(R_{A} \cdot \mathrm{MeV}^{2} / M_{\mathrm{Pl}}\right)^{1 / 9} \quad \text { or } \quad h_{A} \gtrsim\left(R_{A} \cdot \mathrm{MeV}^{2} / M_{\mathrm{Pl}}\right)^{1 / 17}
$$

respectively. If for example $m_{s} \sim 10^{10} \mathrm{GeV}$ and $N_{A}^{1 / 4} \sim 10$, we need

$$
h_{A} \gtrsim 10^{-4} \text { or } h_{A} \gtrsim 10^{-2}
$$

respectively. If we want to incorporate $\mathrm{TeV}$ baryogenesis in the $\mathrm{S}$-throat, then we need

$$
h_{A} \gtrsim 10^{-2} \text { or } h_{A} \gtrsim 10^{-1}
$$

respectively. These are reasonable for an A-throat. Note that for the 5-dim RS setup, the bound is much looser.

The KK modes tunneled into the S-throat will become thermalized in a rate

$$
\Gamma_{K K}\left(t_{\text {tun }}\right) \approx n\left(t_{\text {tun }}\right) \sigma_{K K} \approx\left(h_{S} R_{S}^{-1}\right)^{-\frac{\gamma}{\gamma+4}}\left(M_{\mathrm{Pl}} \Gamma_{\text {tun }}\right)^{\frac{2(\gamma+3)}{\gamma+4}} \sigma_{K K}
$$

where the time dependence of the number density of the KK modes $n(t)$ is obtained from formula same as Eq.(6.1) and (6.3). As long as this rate is larger than the tunneling rate, which is similar to the Hubble expansion rate at the end of the tunneling, we can assume the thermal equilibrium. This requirement translates into a condition on $h_{S}$ which is easy to satisfy. ${ }^{5}$

Since the warp factor of the S-throat is much smaller than that of the A-throat, the KK spectrum is much denser. After the thermalization, the KK modes will decay to the much lower mass levels and become much more difficult to tunnel back out. Therefore the tunneling from $\mathrm{A}$ to $\mathrm{S}$ is effectively a one-way process. This streaming of the KK modes heats the S-throat. At the end of the tunneling process, the universe will become dominated by the thermalized KK modes in the S-throat and become radiationdominated again.

\footnotetext{
${ }^{5}$ Ignoring all other factors, this condition requires the warp factor $h_{S}<\Gamma_{\text {tun }}^{\frac{\gamma+2}{5 \gamma+16}} \cdot \operatorname{mass}^{-\frac{\gamma+2}{5 \gamma+16}}$. For example, according to the discussion in Sec. 8.2 and the result (8.5), this is naturally satisfied.
} 
But there is a subtlety here. Due to the same reason, i.e. the S-throat has a much smaller warp factor, the initial thermalized gas may become stringy. To get an estimate on the temperature using the KK spectrum (6.2), after tunneling we have

$$
\frac{M_{\mathrm{Pl}}^{2}}{t_{\text {tun }}^{2}} \approx \frac{T_{S}^{\gamma+4}}{\left(h_{S} R_{S}^{-1}\right)^{\gamma}} \text {. }
$$

Borrowing the result (8.6) from Sec. 8, we see that

$$
T_{S}\left(t_{\text {tun }}\right) \lesssim h_{S}^{\frac{\gamma+2}{\gamma+4}} N_{S}^{\frac{1}{\gamma+4}} M_{\mathrm{Pl}}^{\frac{2}{\gamma+4}} R_{S}^{-\frac{\gamma+2}{\gamma+4}} \propto h_{S}^{\frac{\gamma+2}{\gamma+4}} m_{s}
$$

On the one hand, we see that this upper bound is typically much smaller than the mass level $h_{A} R_{A}^{-1}$ of the lowest $\mathrm{KK}$ mode in the A-throat; on the other hand, we see that it is possible for $T_{S}\left(t_{t u n}\right)$ to exceed the red-shifted string scale $h_{S} m_{s}$ in the S-throat. To proceed, we will ignore the involvement of the possible string states for the following reasons. First, as universe cools down the thermalized gas will eventually become a gas of KK particles. So the estimates on the KK relics still apply. Second, in the most pedagogical sense, since $(\overline{6.16})$ is just a upper bound, we may adjust various parameters to make $T_{S}\left(t_{\text {tun }}\right)$ stay below the red-shifted string scale. However, a better understanding of such a possible stringy phase and its consequences is clearly very interesting, because it may not happen too early away from the BBN.

Before continuing our story in the S-throat, we note that there is another possible period during the tunneling that deserves attention. As we have seen from Sec. 2, if bulk resonance happens, KK will stay in the bulk in this intermediate period, so it is also important to analyze the graviton production and possible KK relics in this case. It is easy to check that any $\mathrm{KK}$ in this resonance period is decoupled from each other. So any stable KK, once produced through decay process, will be a problem. But this is not a big concern, because there is usually no good candidates for stable KK modes in the bulk. The wavefunction of these KK modes spread over the bulk, and will always hit some throats. This is in fact how we estimated the tunneling rate to X-throat in Sec. 2.2. For graviton production, we integrate all particles and get a very small ratio

$$
\frac{\rho^{\text {grav }}}{\rho^{\text {tot }}} \approx g^{1 / 4} M_{\mathrm{Pl}}^{3 / 2} \sigma_{K g} \Gamma_{t u n}^{1 / 2},
$$

where $\Gamma_{\text {tun }} \sim h_{A}^{9} R_{A}^{-1}$ is the tunneling rate from the A-throat to the bulk.

\subsection{KK evolution in S-throat}

The evolution of KK modes in S-throat is similar to that in A-throat. A small difference is the additional relativistic degrees of freedom $g_{0}$ due to the Standard Model particles at the end of the mass spectrum. So we take $g$ to be $n_{K K}^{\gamma}$ if $g>g_{0}$, and approximately constant $g_{0}$ later on. 
The relative abundance of the possible KK relics is similarly obtained,

$$
\left(\frac{\rho_{d e c}^{K K}}{\rho^{t o t}}\right)_{R D M D} \approx \sum_{n_{K K}} g^{-1 / 4} M_{\mathrm{Pl}}^{-1} \rho_{R D M D}^{-1 / 4} \sigma_{K K}^{-1}
$$

Comparing to (6.7) this is typically negligible since $\sigma_{K K}$ here is enhanced by powers of $h_{S} \ll h_{A}$.

The graviton is mainly produced around $t_{t u n}$,

$$
\left(\frac{\rho^{\text {grav }}}{\rho^{\text {tot }}}\right)_{B B N \text { or } R D M D} \approx \tilde{\alpha}_{S}^{1 / 4}\left(h_{S} T_{3}^{1 / 4}\right)^{-\frac{\gamma}{\gamma+4}} M_{\mathrm{Pl}}^{\frac{2 \gamma+6}{\gamma+4}} \Gamma^{\frac{\gamma+2}{\gamma+4}} \sigma_{K g} .
$$

For small $\gamma$, this ratio is much smaller than (6.9) due to the dilution of the KK particles during the tunneling.

Overall, we summarize the graviton and KK abundance obtained in this section and Sec. 4 for the double throat case.

For the graviton, the total abundance is a summation of those from the initial decay in Sec $1,\left(\frac{R}{L}\right)^{6} h_{A}^{2}\left(\frac{t_{A n r}}{t_{t u n}}\right)^{2 / 3}$ (where the last extra factor is added due to the matterdominated phase during the tunneling), the annihilation in the A-throat Eq. (6.9), the S-throat Eq. (6.19), and the bulk Eq. (6.17) if resonance happens. All of them are suppressed by various combinations of warp factors, bulk size and matter-dominated phase, so easily become very small. The actual value is parameter dependent.

For the KK relics, Eq. (6.18) is suppressed by powers of the S-throat warp factor, so the A-throat contribution (6.7), if stable, is typically larger. Evaluating (6.7) using (3.19) and (6.4), we get

$$
\Omega_{R D M D}^{K K} \sim\left(\frac{R_{A}}{L}\right)^{6} h_{A} M_{\mathrm{Pl}}^{3 / 2} \rho_{R D M D}^{-1 / 4} R_{A} \Gamma_{\text {tun }}^{1 / 2} .
$$

For example, if $M_{\mathrm{Pl}} \approx 10^{18} \mathrm{GeV}, \rho_{R D M D}^{1 / 4} \approx 1 \mathrm{eV}, m_{s} \approx 10^{10} \mathrm{GeV}$ and $N_{A} \approx 10^{4}$, for the resonance case we need

$$
\left(\frac{R_{A}}{L}\right)^{6} h_{A}^{5.5} \lesssim 10^{-31}
$$

to satisfy $\Omega_{R D M D}^{K K} \lesssim 1$. This for example can be satisfied if $L \approx 10^{3} R_{A}$ and $h_{A} \approx 10^{-3}$. Note that the resonance condition typically requires a large $L$. For the non-resonance case, we need

$$
\left(\frac{R_{A}}{L}\right)^{6} h_{A}^{9.5} \lesssim 10^{-31}
$$

This for example can be satisfied if $L \approx 10^{2} R_{A}$ and $h_{A} \approx 10^{-2}$. 


\section{The bulk case}

Before going to the more general multi-throat case, let us look at the case where either SM branes or brane annihilation are in the bulk.

So far we have considered SM branes in throats. These throats can be regarded more generally as black hole like geometry, created by large number of D3-branes, 3-form fluxes or even induced large D3-charges on the D7-branes. To enter these geometries KK modes need to tunnel. But it is also possible that some small number of branes appears in the bulk rather than hiding in some throats. For these branes, the warping they induce is small so KK modes do not have to tunnel a significant barrier. In this situation, if the KK wavefunction appears in the bulk, it can intersect with these branes and the KK particles can decay into light brane fields. So they start to heat the bulk branes instead of heating a throat through tunneling. This can happen in the bulk resonance case when KK propagate in the bulk, or in the non-resonance case where KK also has a wavefunction in the bulk although it is not propagating. Namely these small number of branes in the bulk may become SM branes.

The heating in this section can be easily studied by collecting a few results in Sec. 5 and 6. There are three possibilities.

We first discuss the case in which the brane annihilation still happens in a throat (A-throat). The heating of the SM branes is caused by the KK particles tunneled out of the A-throat. The analyses in the A-throat are the same as that in Sec.6.1. The difference is that, the KK particles will tunnel to the bulk and decay to lower KK modes and SM brane fields. Same as we discussed at the end of Sec.6.2, any stable KK will be dangerous in this case, but the requirement of no stable $\mathrm{KK}$ is reasonable for the bulk since they will always intersect with the bulk SM branes and decay. The graviton production is small and the same as (6.17). So the thermal history is rather similar to the double throat resonance case and the heating works.

The second case is when both brane annihilation and SM branes are in the bulk. The analyses for the KK modes are the same as in the single throat case. We get the same expressions as in Eqs. (5.10) and (5.14). But in this case, there is no longer warp factor and bulk size enhancement factors to the KK interactions, $\sigma_{K K} \approx M_{\mathrm{Pl}}^{-2}$ and we get

$$
\Omega_{R D M D}^{K K} \approx \sum_{n_{K K}} g^{-1 / 4} M_{\mathrm{Pl}} \rho_{R D M D}^{-1 / 4} .
$$

However we still do not worry about the KK relics in the bulk, because they are unstable against decaying into brane fields. The graviton production during the KK evolution is still very low. Overall, for this case, the main graviton abundance problem comes from the initial brane and string decay period discussed in Sec. Ref. [36] shows that in flat space the graviton is produced more than KK particles in the initial string decay. In all other cases that we considered, the brane annihilation happens 
in a throat so this graviton problem is absent. This is the only case that can have a serious problem in heating. This problem is present in models such the branes-at-angle scenario [56], the D3-D7 inflation with D7-branes in the bulk [57], or the D3-anti-D3brane scenario in the bulk [2, 3], if the SM branes are also in the bulk.

This graviton problem can be cured if there is a matter-dominated era before the BBN, so the graviton density can be considerably red-shifted. This leads to the third case where we locate the SM branes in an S-throat, although the brane annihilation happens in the bulk. If there is no extra small number of (anti)branes left in the bulk, the KK modes will eventually tunnel into the S-throat. This introduces a matter dominated phase similar to what we have seen in the double throat case. The graviton problem is hence cured by the red-shift and the story of the S-throat in Sec. 6.3 applies here.

\section{Multi-throat heating}

In this section, we consider the heating in a more general multi-throat compactification. In this case, the brane-anti-brane annihilation at the end of the inflation still happens in the A-throat. The heating from the A-throat to the bulk or the S-throat follows the same analyses in the previous section, but the presence of many other throats gives additional interesting phenomena.

\subsection{Throat deformation in Hubble expansion}

The spectrum in a throat is mainly determined by its minimum warp factor. However in the Hubble expansion background, the warping of a throat cannot be arbitrarily small. Even if we start with an infinitely long throat, the Hubble energy will impose an infrared cutoff on the warp factor.

This Hubble back-reaction may be analyzed in different ways [58, 12, 37, 59]. One way to look at it is to consider the back-reaction of the closed string created in the IR side of the warp space [12]. Assuming an infinitely long throat, such string creation will be inevitable because the local string scale will be red-shifted down the throat and eventually fall below the Hubble energy scale. The energy density of these strings is $H^{4}$. The back-reaction of such closed strings become significant to the source of the throat if

$$
H^{4} \approx h_{\min }^{4} N T_{3}
$$

since the throat with $R^{4} \approx N / T_{3}$ can be thought of being sourced by $N$ number of branes. Therefore, although in static background we can have multiple throats with arbitrary warp factors, in the Hubble expansion background they will have a cutoff at

$$
h_{\text {min }} \approx \frac{H R}{\sqrt{N}}
$$

if they are too long. 
Another way to look at it is to consider various moduli that stabilize the throat [37. Although in static background, throat with arbitrary warp factors can be constructed by stabilizing those moduli, the moduli mass will obtain a correction in a Hubble expansion background, and therefore cause the deformation of the throat. The explicit evaluation along this line has so far been difficult due to the subtleties analyzing the supergravity equations in the presence of small warp factors [37, 59].

Here we give a toy model of this type to roughly estimate this effect. Consider an infinitely long throat generated by $N$ stack of branes rather than fluxes. In the Hubble expansion background, the brane will fluctuate in the transverse directions,

$$
\Delta \phi \approx \sqrt{T_{3}} \Delta r \approx \frac{H}{2 \pi} .
$$

This fluctuation causes a deviation of the brane moduli which otherwise are located exactly at the origin. So the throat is no longer extended to infinity at $r=0$ but terminated at a minimum $r_{\min } \approx \Delta r$. This corresponds to a minimum warp factor

$$
h_{\min } \approx \frac{\Delta r}{R} \approx \frac{H R}{\sqrt{N}} .
$$

We see that in this toy model we get a consistent picture as in Eq. (8.2). This consistency can be regarded as a duality relation between deformed AdS and CFT: Eq. (8.2) is obtained from the point of view of the gravity side, while Eq. (8.4) the field theory side.

These analyses apply to both the inflationary and heating epoch, and give a timedependent minimum value for throat warp factor at corresponding moment. As a remark, we note that the energy associated with those closed strings and moduli potentials is negligible to the heating energy, since it is of order $\mathcal{O}\left(H^{4}\right)$ and much less than the inflationary energy. But it can create a spectrum of cosmic strings with relatively low tension [12].

\subsection{Selection of long throats}

In the presence of many throats, the tunneling of KK modes can have many channels. That is, it is possible for the KK modes to go from A-throat to different throats. So which throats become dominantly heated depends on the tunneling branching ratio.

A necessary condition for the tunneling discussed in Sec. 2.2 and 2.3 to happen is the matching of energy levels in both throats. This condition requires that an energy eigenvalue in the $\mathrm{X}$-throat fall within the energy width of the initial state in the A-throat. The width of the lowest KK mode in the A-throat is determined by its tunneling rate $\Gamma_{\text {tun }}$, as one can see from the uncertainty principle. So, the more finely spaced is the X-throat spectrum, the more likely such a matching can be found. ${ }^{6}$ A long throat is thus

\footnotetext{
${ }^{6}$ Note that this is different from the argument that the large phase volume in a long throat guarantees more heating after string decay because of equipartition, as discussed in Ref. 34, 35. As pointed out in the same references, the presence of the potential barrier invalidates such arguments. Here even for a short throat, if a mass level matching is accidentally satisfied, the tunneling will still proceed (but may oscillate). Decreasing warp factor increases this chance.
} 
favored during the tunneling heating. Since in a throat with a minimum warp factor $h_{X}$, the energy spacing for the s-wave is $h_{X} R_{X}^{-1}$, if

$$
h_{X} \lesssim \Gamma_{\text {tun }} R_{X},
$$

the tunneling will generically happen for this throat. The Hubble constant during the

tunneling is $H \approx \frac{1}{t_{t u n}} \approx \Gamma_{t u n}$, so the allowed minimum warp factor (8.2) in this Hubble expansion background is

$$
h_{m i n} \approx \frac{\Gamma_{t u n} R_{X}}{\sqrt{N_{X}}} .
$$

So the condition (8.5) is allowed.

Thus we have seen a dynamical mechanism of selecting a long Standard Model throat in the multi-throat heating. For example, for the tunneling rate (2.32), if roughly $R_{A} \sim R_{X} \sim D, \Gamma_{\text {tun }} \sim h_{A}^{9} D^{-1}$, any throat with

$$
h_{X} \lesssim h_{A}^{9}
$$

will be generically heated, while heating probability for a shorter throat is smaller. A throat with a large hierarchy ratio $\left(\sim 10^{-10}\right)$ in the sense of Randall and Sundrum can be naturally selected in this way. If the SM branes are in the bulk, such a mechanism will favor a large bulk size.

As a comment, we note that, even if the mass levels between the A and X does not match, tunneling can proceed through a secondary effect. A virtual KK particle is first created in the X-throat, and then splits into several lower mass states such as lower KK modes or brane fields. The kinetic energy of these light particles can compensate the mass level difference. It will be interesting to estimate the branching ratio of such a process and we leave it for future study.

\subsection{Warped KK dark matter and hidden dark matter}

In this subsection we discuss two interesting possibilities of non-relativistic dark matter. The first is the KK relics in the S-throat. In our setup, the lowest KK mode in the S-throat will interact with the SM branes and decay into brane fields, i.e., SM particles as light open string modes. However, higher level KK modes can have a long enough life time, e.g., if they are associated with a conserved angular momentum in the throat. For example, in the Klebanov-Strassler throat, besides the excitation quantum number $n$, the KK modes can also have angular quantum numbers $j$ for the approximate $S^{3}$ and $l$ for $S^{2}$ in a realistic throat, i.e., a warped deformed conifold. The (approximate) $S^{3}$ remains finite at the bottom of the throat (a property of the deformation of the warped conifold). As a consequence, $j>1$ quantum number costs less energy than the $l$ quantum numbers [40]. The KK modes with only $j$ quantum numbers have non-vanishing wave functions at the bottom of the throat, so they can easily decay to SM particles. (Recall 
that transverse momenta can be absorbed by the solitonic-like branes.) On the other hand, the $S^{2}$ shrinks to zero at the bottom of the throat, so the wave functions of $l>0$ KK modes are suppressed at the origin, much like that in the hydrogen atom. Having no overlap with the SM branes, the light $l>0$ modes will not decay to SM particles. They couple to SM particles via the exchange of a s-wave $\mathrm{KK}$ mode, so two $l>0 \mathrm{KK}$ modes can annihilate. However, this annihilation rate drops rapidly as the universe expands, and these KK relics abundance can be estimated as we did for the simplified throat in the previous sections. Their tunneling rate is very small due to the smallness of the S-throat warp factor and additional non-s-wave suppression factors. So these lightest angular KK modes are quite stable.

Such a non-relativistic relic was a concern in Ref. 355 since their abundance was estimated to be extremely large. So if stable they would over-close the universe. In the previous sections, we have analyzed the KK thermal evolution and the abundance of these KK relics more carefully. As we discussed, for example in Eq. (5.5), these relics typically decouple non-relativistically. From Eq. (5.11) and (6.18), we can see that the relative density of these KK relics is suppressed by warp factors, bulk size and matterdominated tunneling phase, and so can be naturally made safe. (To compare with the analysis in Ref. [35], we note Eq. (4.16) in [35] is based on relativistic decoupling and has no adjustable small parameters. Here Eq. (5.11) and (6.18) have several important differences: a factor of $h_{A, S}^{2}$ due to the non-relativistic decoupling; a factor of $(R / L)^{6}$ from the throat-versus-bulk effect; and in the double throat case, an extra suppression $\left(t_{\text {Anr }} / t_{\text {tun }}\right)^{1 / 2}$ for the A-throat from the matter-dominated tunneling phase.)

Of course, one can then tune various parameters in the formulae to make them just account for the density of the observed dark matter. Namely they can become the warped KK dark matter. In the single throat case, the relics abundance is given by (5.11); in the double throat case, (6.18) is typically smaller than (6.7), but it may become important if the KK relics in A-throat have too short (tunneling or decay) lifetime. (Otherwise we will discuss it in Sec.9.2.) They may be detected by collider experiments or underground dark matter searches. For example, when two SM particles collide at the red-shifted KK energy scale, they can produce an intermediate unstable s-wave KK particles, then subsequently decay into two warped KK dark matter particles. They will have different cross-sections than those of the more standard dark matter candidates. Discussions on various other kinds of KK dark matter can be found in e.g., Ref. [41, 42, 43, 44, 45].

Next we consider another type of dark matter. In the multi-throat setup, when the KK particles are tunneling from the A-throat to heat the S-throat or the bulk SM branes, similar process can also happen for any throat which satisfies the mass level matching condition discussed in Sec. 8.2 and Sec. 2. In particular, such a tunneling happens generically for any throat long enough to satisfy (8.5). Even in the single throat case, it is possible for a tiny amount of KK modes to tunnel out to other throats. ${ }^{7}$ In this

\footnotetext{
${ }^{7}$ The possibility that KK particles may tunnel to other throats is also discussed in Ref. [35, 36].
} 
subsection and Sec. 9.1, we propose that the energy density going into those throats can create new dark matter candidates. Using the knowledge of the thermal evolution that we obtained in this paper, we will discuss the properties and thermal histories of these dark matter candidates and the plausibility of this proposal, in particular paying attention to the dark matter coincidence problem. We call those throats as D-throats, with D stands for dark matter, and call those dark matter as hidden dark matter, because they are in a hidden sector and their wavefunction has exponentially small overlap with our visible sector.

The identities of the hidden dark matter in D-throats can be very different from those of the warped KK dark matter. For a D-throat without any branes in the end, as in Sec. 6.1, the KK tower will eventually be lowered to the ground state. So the hidden dark matter in such a throat will be the lowest KK particles, but not those with conserved angular momentum. These KK particles are mutually decoupled and cannot find each other to annihilate. Different from Sec. 6.1, since the D-throat has a much smaller warp factor than the A-throat, these lowest KK modes take much longer to tunnel out and therefore become stable. For a D-throat with branes in the end, the identity of the hidden dark matter can be either similar to that of the warped KK dark matter in the S-throat, or more exotic particles analogous to our baryons.

These dark matter have very different properties comparing to the usual dark matter candidates such as the warped KK dark matter mentioned above, the SM KK dark matter [43, 44, 45], axions [60, 61, 62, or the lightest supersymmetric particles (LSP) 63, 65]:

1. Coupling: For the usual dark matter candidate, although they are decoupled to the Standard Model particles at low energy, they can couple if the energy is excited reasonably higher. For example, for the warped KK dark matter in the S-throat, the corresponding energy scale is the warped KK scale, lower than the red-shifted string scale; for the LSP in the MSSM, it is around the supersymmetry breaking scale $\mathrm{TeV}$.

However, for the hidden dark matter in the D-throats, it almost completely decouples from the Standard Model. As we can see from Eq. (2.17)-(2.19), the wavefunction of a KK mode damps at least by a factor of $h_{D}^{8}$ from $\mathrm{D}$ to S-throat. The particles in the S-throat can couple to the hidden dark matter only if they are excited out of the throat, namely by roughly the UV string scale. Hence practically, the only interaction between the hidden dark matter and the Standard Model (or its extension) is through graviton mediation. Of course the hidden dark matter can have self-interactions.

Ref. [35] raised a concern that this would generate KK relics that over-close the universe and destroy the BBN. In Sec. 9.1, we will see how the unusual properties of the hidden dark matter can be used to avoid such problems. Ref. [36] gave a lower bound on these throats to avoid the overheat of the S-throat after BBN due to KK tunneling from those throats. 
2. Thermal history: For the usual dark matter, they initially thermalize with the rest of the particles and closely participate in the thermal history when the temperature in the S-throat is high. Only later on after freeze-out, they evolve independently.

However, the hidden dark matter in D-throats has its own thermal history. They have different temperatures once particles thermalize in the D-throats. The thermal evolutions of the particles in the D-throats and the S-throat are independent, with the net effect driving the spatial expansion of the whole universe.

3. Relic density: For the usual dark matter, the relic density is set by the decoupling condition in the course of the thermal evolution. The decoupling happens when the density of the particles drops low enough so that their annihilation rate is below the expansion rate. After that, they are driven apart forever by spatial expansion and cannot find each other to annihilate. We have seen such an example in the discussions of the KK relics in the previous sections.

But for the hidden dark matter in D-throats, it never evolves in the thermal history of the S-throat and decouples at the beginning as soon as KK particles tunnel to the D-throats and decay. The relics density is set by the initial conditions during the tunneling and the properties of the D-throats.

\section{Hidden dark matter}

In the first subsection, we study more details of the hidden dark matter in D-throats proposed in Sec. 8.3 and the dark matter coincidence problem. In the second subsection, we discuss another type of hidden dark matter in the A-throat and its possible implications on cosmic rays.

\subsection{Hidden dark matter and dark matter coincidence problem}

We first list some interesting consequences and constraints followed from the properties discussed in Sec. 8.3. In this subsection, when we make explicit comparisons, we will choose to compare the hidden dark matter with the LSP dark matter for example. Also we will choose S-throat as the location of SM branes. The discussion still applies if we replace it with SM branes in the bulk.

1. The mass of the LSP has to be greater than $100 \mathrm{GeV}$, because otherwise it would have already shown up in colliders. But this is not the concern for the hidden dark matter. In fact there is little constraint on the mass of the hidden dark matter particles. A more relevant statement is that the hidden dark matter does not have to become non-relativistic before the temperature is $100 \mathrm{GeV}$ in the S-throat. It only has to take effect before the radiation-matter transition epoch in the S-throat at around $1 \mathrm{eV}$. 
2. Each throat, including the S-throat and D-throats, has its own RDMD transition epoch. To be consistent with our observation, the S-throat has the latest RDMD transition. The RDMD transition in D-throats can have different nature, depending on their matter contents. We will give two examples later. For an observer in a D-throat, after its local RDMD transition, she will observe a period of hot dark matter-dominated era, contributed by the S-throat.

3. From the observations, we know that the density of the dark matter is approximately 5 times of that of the baryons. If the hidden dark matter in D-throats accounts for most of these dark matter, we require

$$
\rho_{D}\left(t_{D}^{R D M D}\right) \frac{a^{3}\left(t_{D}^{R D M D}\right)}{a^{3}\left(t_{S}^{R D M D}\right)} \approx 5 \rho_{S}\left(t_{S}^{R D M D}\right),
$$

where the subscript D or S refers to the local quantity of the D or S-throat respectively.

Moreover, at the time of $\mathrm{BBN}$, we require the density (of either matter or radiation) in the D-throats be much smaller than that in the S-throat, so that the process of the BBN is not affected. Therefore during the tunneling heating, the most heated throat will turn out to be the S-throat. This is consistent with the previously mentioned requirement that the RDMD transition in the S-throat happens last. In fact, the energy distribution between the $\mathrm{S}$ and D-throats does not have to be too different. The requirement

$$
\frac{\rho_{D}\left(t_{S}^{B B N}\right)}{\rho_{S}\left(t_{S}^{B B N}\right)} \lesssim \text { a few percent }
$$

will be enough to be consistent with the observations. This variation of the initial densities is due to the variation of the tunneling rate with respect to different throats. Indeed from the formulae (2.31), 2.32) and (8.5), we can see that, while the tunneling rate has the same scaling dependence on the warp factor $h_{A}$, variations are expected for D-throats having different locations, length scales or warp factors.

We now look at two examples with different contents of hidden dark matter in Dthroats. We will focus on the following coincidence problem: why the density of the dark matter happens to be roughly the same as (five times) that of the baryons? For the usual dark matter, this density is formed when the density of the dark matter particles exponentially decreases and eventually freezes out after the annihilation rate drops below the Hubble expansion rate. Then this question amounts to showing whether the required annihilation cross section is naturally obtained given a model. For example, it has been argued that the WIMP may naturally have this cross section. Here, since we have very 
different properties, it will be very interesting to see what new aspects hidden dark matter offers to this classic question.

- Example 1: Imagine a D-throat without any brane located at the tip, so the hidden dark matter will be the lowest $\mathrm{KK}$ mode with mass $m_{D}^{K K} \approx h_{D} R_{D}^{-1}$. This is the case of hidden KK matter.

In this example, the RDMD transition in the D-throat happens when the local temperature crosses $m_{D}^{K K}$ at $T_{D}\left(t_{D}^{R D M D}\right) \approx h_{D} R_{D}^{-1}$. At this moment, the coincidence problem requires the energy density in the D-throat to be five times of the baryons in the S-throat. Since the total energy density is still dominated by radiation in the S-throat until $t_{S}^{R D M D}$, we obtain the ratio of the energy density between the $\mathrm{D}$ and S-throat at and before $t_{D}^{R D M D}$,

$$
\frac{\rho_{D}}{\rho_{S}} \sim 5 \frac{T_{S}\left(t_{S}^{R D M D}\right)}{T_{S}\left(t_{D}^{R D M D}\right)}
$$

Suppose initially we have (9.2), Eq. (9.3) indicates that

$$
t_{S}^{R D M D}>10^{4} t_{D}^{R D M D}
$$

To get a more explicit requirement on the D-throat, we use Eq. (6.2) and find initially

$$
\frac{T_{D}}{T_{S}} \approx\left(\frac{\rho_{D}}{\rho_{S}}\right)^{\frac{1}{\gamma+4}}\left(\frac{h_{D} R_{D}^{-1}}{h_{S} R_{S}^{-1}}\right)^{\frac{\gamma}{\gamma+4}} .
$$

At the local RDMD transition in the D-throat,

$$
T_{D}\left(t_{D}^{R D M D}\right) \approx h_{D} R_{D}^{-1}
$$

At the same time, we know from the condition $(9.3)$ and $T_{S}\left(t_{S}^{R D M D}\right) \approx 1 \mathrm{eV}$ that

$$
T_{S}\left(t_{D}^{R D M D}\right) \approx \frac{\rho_{S}}{\rho_{D}} \cdot 5 \mathrm{eV}
$$

Plug Eq. (9.6) and (9.7) into (9.5), we get

$$
h_{D} \approx\left(\frac{\rho_{S}}{\rho_{D}}\right)^{\frac{\gamma+3}{4}}\left(\frac{R_{S}}{R_{D}}\right)^{\frac{\gamma}{4}}\left(5 \mathrm{eV} \cdot R_{D}\right)^{\frac{\gamma+4}{4}} h_{S}^{-\frac{\gamma}{4}} .
$$

In this example, the coincidence problem results in a tuning of the D-throat warp factor for any ratio $\rho_{S} / \rho_{D}$, as long as the condition (9.4) is satisfied. For the bulk SM branes, one simply replaces $\rho_{S}$ with $\rho_{\text {bulk }}, h_{S}^{-1} R_{S}$ with $L$ in Eq. (9.8).

- Example 2: Perhaps the S-throat does not have to be special in the sense that branes can also be located in other throats such as D-throats as well. In each D-throat, there are different versions of the "Standard Model" on branes. In such a case, the relevant hidden dark matter content is no longer the lowest KK mode, it is different 
matter fields on branes, analogous to our baryons. More importantly, the mass of these matter is no longer of the local KK mass scale. The hidden dark matter is massless at the leading order comparing to this scale, just like the baryons in our Standard Model throat. This is the case of hidden matter.

Again we naturally assume that S-throat and D-throats have similar but variable heating branching ratios (for example, if they all satisfy (8.5)). We pick the most heated throat as the S-throat for reasons discussed above. The thermal evolution of the Dthroats are similar to the S-throat. As the universe expands, the KK tower lowers and eventually transfer its energy to the brane fields. The branes fields are mostly massless particles, but there can be a tiny fraction of asymmetric "baryons" matter generated by "baryogenesis". Since radiation red-shifts faster than matter, the "baryon" matter eventually dominates in the D-throats. What follows is a period of "hot dark matter" dominated era contributed by the S-throat. After that the whole universe becomes matter-dominated.

In this case, the coincidence problem reduces to the similarity between the "baryogenesis" efficiencies on the "Standard Models" in the D-throats and that on the Standard Model in the S-throat. To give an example, we assume initially $\rho_{S} \sim 20 \rho_{D}$. If the Dthroat matter density is similar to ours so that $\rho_{S}^{\text {matter }} \sim 0.2 \rho_{D}^{\text {matter }}$, the RDMD transition happens a little earlier in the D-throat - by a factor of 100 in terms of the scale factor. That is, Eq. (9.4) still applies here. By the time that the RDMD transition happens in the S-throat, we have both the baryons and hidden matter with similar abundance. Both the mass of the hidden dark matter and its final abundance become much more independent of the D-throat warp factor.

Both examples give very interesting illustrations of how hidden dark matter can behave differently against the usual intuition. Naively, if the energy density that goes into a dark matter candidate (e.g. LSP) is as large as a few percent of that goes into the SM particles, it surely over-closes the universe and destroys the BBN. This is because the LSP dark matter candidates become non-relativistic before the temperature is $100 \mathrm{GeV}$. In terms of time, this happens more than $10^{22}$ times earlier than the RDMD transition. Only extremely tiny fraction of energy is allowed to deposit into the dark matter to account for such a large red-shift. But hidden dark matter can become non-relativistic much later, as late as in (9.4) - reducing $10^{22}$ to $10^{4}$ - which greatly shortens the redshift. This is achieved, in the first example, by having a long D-throat and therefore very low KK levels; in the second example, by having similar amount of matter and radiation between the hidden sector and the visible sector.

\subsection{Hidden dark matter and cosmic rays}

So far in this section, we have studied the properties of the hidden dark matter lying in D-throats which are different from both $\mathrm{S}$ and A-throat. But in the double throat case or the bulk case, the KK modes with specific conserved angular momentum in the 
A-throat can also become viable dark matter candidates. It is possible that these KK modes have very small tunneling rate. For example, the s-wave in the A-throat have a tunneling rate $h_{A}^{9} R_{A}^{-1}$ or $h_{A}^{17} R_{A}^{-1}$. This s-wave is responsible for the tunneling heating. For a $l$-th partial wave, the tunneling rate is expected to be reduced by a factor of $h_{A}^{4 l}$ for the simplified geometry studied in Sec. 2 (same as the absorption rate in [53]). Hence a reasonable higher partial wave can be stable against the tunneling. If this angular momentum is also approximately conserved (now it can be associated with either $S^{3}$ or $S^{2}$ in the KS throat since we do not have branes left in the A-throat), it becomes a candidate of stable relics. We have estimated the relics abundance of such KK modes in Eq. (6.7). We see that it again contains various tunable small parameters and can be a viable dark matter candidate.

An interesting feature of this dark matter is that its properties lie in between the hidden dark matter in D-throats and the warped KK dark matter in S-throat. In terms of couplings, it is similar to the former - it only couples to the visible sector through gravity and usually cannot be produced in colliders. So it is still the hidden dark matter. But in terms of the properties of the thermal history, it is more like the latter - it decouples as a non-relativistic thermal relics and its abundance can be calculated in the usual way.

We have been familiar with the fact that large cosmic strings can be left in the A-throat as a result of closed string evolution. For these cosmic strings, they cannot tunnel. The evolution of cosmic strings will lead to a scale-invariant spectrum (i.e., cosmic strings will come in all sizes in some distribution). In general, that will yield a density depending on string tension $\mu$. It goes like $G_{N} \mu \Gamma$ of the critical density, where $\Gamma$ is a numerical number that is around 10 to 100 [64]. With $G_{N} \mu<10^{-6}$, large cosmic strings contributes very little to the critical density. But now we see that it is also possible that the closed string and KK evolution lead to some stable KK modes in the A-throat. Then those KK modes can have a sizable density to contribute to the dark matter.

Such a dark matter is typically very heavy. Take the double throat non-resonance case as an example. In the numerical example of Eq. (6.22), the mass of this hidden dark matter is

$$
M_{D M} \sim 10^{7} \mathrm{GeV} .
$$

One can easily change the string scale since the bound $\Omega_{R D M D}^{K K} \lesssim 1$ on (6.20) is not very sensitive to this variation. For example, taking $m_{s} \approx 10^{13} \mathrm{GeV}, N_{A} \approx 10^{2}$ and still keeping the tunneling rate $\Gamma_{\text {tun }} \sim h_{A}^{17} R_{A}^{-1}$ and the other parameters same, we get $h_{A} \sim 10^{-2}$ and $L \sim 60 R$ to saturate the bound. In this example, the mass of the hidden dark matter becomes

$$
M_{D M} \sim 10^{11} \mathrm{GeV} .
$$


There are many interesting consequences of such a dark matter.

First, we have seen that this hidden dark matter belongs to thermal relics, so both (9.9) and (9.10) evade a well-known bound [66] which states that the maximum mass of a dark matter is a few hundred $\mathrm{TeV}$ if it is a thermal relic. This bound comes because larger dark matter mass leads to smaller annihilation cross section and more red-shift, and therefore excessive dark matter density. The reason that we evade it is the extra matter dominated tunneling phase discussed in Sec. 6.1 and 6.2. During this phase the total energy of the universe, including that of this dark matter, is hung up in the Athroat in a long matter-dominated era. The radiation-dominated phase in the S-throat is therefore much delayed. This greatly increases the allowed ratio between the density of the dark matter and the rest.

Second, this dark matter can have a lifetime longer than the age of the universe, but occasionally tunnel to the visible S-throat. Namely this becomes a novel example of an ultra heavy thermal relic being the source of the ultra high energy cosmic rays. To estimate the lifetime of those angular KK modes in the A-throat, we use the ansatz

$\tau \sim h_{A}^{-17-4 l} R_{A}$. In the above example (9.10), we can see that the lifetime is longer than the age of the universe $10^{17} \mathrm{~s}$ if $l>2$.

Third, since this cosmic ray is due to the decay of the dark matter in our galaxy, they do not have to travel a long distance between galaxies to lose energy through interactions with CMB. So the GZK cutoff [67, 68] does not apply. The energy of these ultra high energy cosmic rays can be as large as $10^{20} \mathrm{eV}$ such as that in Eq. (9.10). Since the source of such decay is not local objects, the distribution of the cosmic rays is more isotropic. Comparing to the distribution of the dark matter annihilation, which is proportional to $\rho_{D M}^{2}(\mathbf{x})$, these cosmic rays will have a distribution proportional to $\rho_{D M}(\mathbf{x})$. Moreover, the energy of the cosmic rays $M_{D M}$ correlates with the inflation scale $V_{\text {inf }}$ and the tension of the cosmic strings $\mu_{F}$,

$$
M_{D M} \approx n_{K K}\left(g_{s} N_{A}\right)^{-1 / 4} \mu_{F}^{1 / 2} \approx n_{K K} N_{A}^{-1 / 4} V_{i n f}^{1 / 4} .
$$

If $N_{A}^{1 / 4}$ is not too big, they are all close to each other in terms of order of magnitude. So in this scenario a measurement of the energy of the relevant cosmic rays predicts the primordial gravitational wave and the cosmic string tension.

Fourth, the nature of this decay can be extremely stringy. This happens in the double throat case. Although the original KK mode in the A-throat is a field theory mode, after it tunnels to the S-throat, its mass is way above the local red-shifted string scale due to the large warping of the S-throat. So detailed observations of such decays may become a useful laboratory to compare with the theoretical calculations in string theory.

\subsection{Comparison to other dark matters}

Besides the LSP that we have discussed, in this subsection, we give a couple of more comparisons between other dark matter candidates and the two main categories of dark 
matter candidates proposed here, i.e. the warped KK dark matter and the hidden dark matter.

The warped KK dark matter shares common features of most usual dark matter candidates, such as their thermal properties and coupling to SM particles. For example, conceptually it is similar to that proposed in the 5-d RS geometry [41, 42]. Besides the differences caused by those between the 5-d RS and 10-d GKP setup, there is no graviton KK in 5-d stable against decaying on the IR branes, so in 41, 42, such a stable particle was put in by hand ("bulky"); here we have a natural and viable realization of their "bulky".

The hidden dark matter is more unusual. Our definition of the hidden dark matter is that they are in a hidden sector, and their wave-function only have an exponentially small overlap with our visible sector. As far as detailed properties are concerned, they may have overlaps with various other kinds of dark matter candidates. But they also have differences. For example, superWIMPs or GIMPs (super-weakly or gravitationally interacting massive particles) such as gravitino or graviton KK modes in universal extra dimension (UED) also have gravitationally suppressed interaction with other particles [69]. But these particles, for example the gravitino, can be the decay product of a supersymmetric Standard Model particles with a mass such as $1 \mathrm{TeV}$. Hence superWIMPs actually couple stronger than the hidden dark matter (but weaker than the warped KK dark matter in the S-throat). For example, if one collides two SM particles at $1 \mathrm{TeV}$, they can become an intermediate supersymmetric particles, through a relatively strong coupling, and then decay to gravitino, through gravitationally weak coupling. But for hidden dark matter, even if the energy of the colliding particles is of order their mass level, they still can only be created through the mediation of gravitons. Because of the same reason, the gravitino can inherit the usual relics thermal abundance of the mother particle, but hidden dark matter in the D-throats has a completely independent thermal history. As another example, there is a proposal of having ultra high energy cosmic rays as decay products of non-thermal heavy dark matter [70]. But as we have discussed, our heavy hidden dark matter in the A-throat is thermal relics, while the non-thermal hidden dark matter in the D-throats does not have to be heavy at all. Of course, more detailed study and comparison with various dark matter candidates is necessary and interesting.

\section{Conclusions and discussions}

In this paper, we have studied the properties of the graviton KK modes in the single and multi-throat compactification, including their wavefunctions, tunneling properties and interactions. We have studied the thermal history after the brane annihilation at the end of the brane inflation, to test whether the heating is compatible with the standard big bang nucleosynthesis. We have found that, as long as the brane annihilation and the SM branes are not both in the bulk, the heating process is viable no matter 
where they are: one of them in the bulk, in the same throat or in different throats, although different scenarios have different thermal histories. The KK thermal relics and the graviton abundance are suppressed by various small factors: warp factors, throat versus bulk size, and the red-shifting factor during the tunneling period. The long tunneling phase required in some cases is not only compatible with a viable heating, but also gives rise to several interesting phenomena: an extra suppression for the KK relics, a dynamical mechanism of selecting a long Standard Model throat, and the generation of hidden dark matter. Our results on the KK thermal history and relics abundance show that there are three types of new dark matter candidates: the warped KK particles with specific angular momentum in the S-throat; the hidden dark matter in D-throats; and the hidden KK particles with higher partial wave in the A-throat. Physically they differ from each other in terms of either the relics thermal history or couplings to the visible sector. The novel properties of the hidden dark matter have especially interesting implications on the dark matter coincidence problem and ultra high energy cosmic rays.

We have seen several possibilities that stringy excitations are produced: in the Athroat during the initial brane decay, in the S-throat after the tunneling from A to S, in any throat with too small warp factors. In the first two cases, the energy density in the stringy excitations can dominate the total energy density initially and then lose it to lower KK modes. In the last case, it never dominates. It will be interesting and challenging to understand if there is any novel effects associated with these phases, besides the cosmic strings discussed in Ref. [19, 20, 21, 22]. For example, it is entirely possible to produce some relatively long lived massive open string modes in the SM branes. These modes will evolve as $a^{-3}$ for a while, eventually decaying to the light SM particles. They tend to enhance the SM entropy contribution during BBN. However, without an explicit model, it is difficult to assess the importance of this possibility. Instead, we see that the graviton KK modes play that role beautifully, but in a way that is more intricate. Here, the important role played by the warped geometry cannot be over-emphasized.

The graviton KK modes in different throats are prime candidates as dark matter. The stable KK modes in the S-throat have qualitatively similar properties as usual dark matter candidates, but with different production and scattering cross-sections. On the other hand, the KK modes in the D-throat or A-throat away from the SM branes have rather different properties. Since they do not interact with SM particles or their superpartners except via gravity, collider experiments and/or underground dark matter searches are useless. One way to detect the hidden dark matter is via gravitational lensing [71]. With better CMBR data, one may fix the parameters in brane inflation more precisely. Together with structure formation simulations, rotational curves etc., they should allow us to better determine the KK spectrum and interactions, their production and subsequent evolution as well as properties of the D or A-throat. One can then check to see if their distribution in our universe matches the lensing observations expected in the near future. 
It is quite possible that the hidden dark matter is only a component of the total dark matter expected. If direct detection of dark matter candidates and their subsequent measurements cannot account for all the dark matter expected, this may be taken as an indirect evidence of hidden dark matter. Violation of the GZK bound on cosmic rays can be a different piece of evidence.

The UV DBI inflation [9, 10] and some special case of the IR DBI inflation 11, 12, require relativistic brane collision as heating, in addition to or as an alternative to the brane annihilation. While we expect the main thermal history after thermalization to be similar to one of our cases here, it is interesting to see how the details may differ especially at the initial moment [72].

In this paper, we try to give an overall picture of heating towards the end of brane inflation. It is an interesting story all by itself. In doing so, we have made only orderof-magnitude estimates and neglected all the numerical factors. A more precise analysis will probably increase the likeliness of some scenarios and decrease that of others, so many interesting aspects are worthy to be studied in more details. It is clear that we have considered only some of the simplest scenarios. There are variations of the scenarios considered here that deserve investigations.

\section{Acknowledgments}

We like to thank Jim Cline, Pier Stefano Corasaniti, Hassan Firouzjahi, Min-xin Huang, Lam Hui, Lev Kofman, Peter Langfelder, Louis Leblond, Kaya Mori, Maxim Perelstein, Sash Sarangi, Sarah Shandera, Jan Pieter van der Schaar, Gary Shiu, Bret Underwood, Ira Wasserman, Erick Weinberg and Haitao Yu for very help discussions. We thank the center of mathematical science of Zhejiang University for the hospitality. XC thanks the Newman Laboratory for Elementary Particle Physics in Cornell University and Aspen center for physics for the hospitality. The work of XC is supported in part by the U.S. Department of Energy. The work of SHT is supported by the National Science Foundation under grant PHY-0355005.

\section{A. a KK cannot decay to all gravitons}

In this appendix, we give a general argument that a KK particle cannot decay to all gravitons, generalizing that in Sec. 3 .

The 10-dim metric contains a warped four large dimensions and six internal dimensions,

$$
\begin{aligned}
d s_{10}^{2} & =g_{\mu \nu}(x, y) d x^{\mu} d x^{\nu}+g_{m n}(y) d y^{m} d y^{n} \\
& =A^{-1 / 2}(y) \hat{g}_{\mu \nu}(x, y) d x^{\mu} d x^{\nu}+g_{m n}(y) d y^{m} d y^{n}
\end{aligned}
$$

The warp factor $A^{-1 / 2}(y)$ and the internal metric $g_{m n}(y)$ is generated by the matter such as fluxes and branes. The wave-functions of KK modes have non-trivial dependence on 
the internal coordinates, $\hat{g}_{\mu \nu}=\eta_{\mu \nu}+h_{\mu \nu}^{K K}(x, y)$, while that of the graviton does not depend on the internal coordinates, $\hat{g}_{\mu \nu}=\eta_{\mu \nu}+h_{\mu \nu}^{g r a v}(x)$. The 10-d action is

$$
S_{10}=M_{(10)}^{8} \int d^{10} X \sqrt{-g_{(10)}} R_{(10)}+S_{\text {matter }}
$$

To get the n-point coupling, we perturbatively expand the action (A.2) to the polynomial of $h_{\mu \nu}$ and $\partial_{\rho} h_{\mu \nu}$. To examine the amplitude of one KK mode coupled to any number of gravitons, we replace one of $h_{\mu \nu}$ with the wave-function of the KK mode in all possible way in each term. We then factor out the wave-function $A^{-1 / 2} h_{\mu \nu}^{K K}$, integrating by parts if necessary. This procedure is the same as what we do to get the equation of motion, except that we now replace $\delta g_{\mu \nu}$ with $A^{-1 / 2} h_{\mu \nu}^{K K}$. So we get

$$
S_{10} \supset-M_{(10)}^{8} \int d^{10} X \sqrt{-g_{(10)}}\left(A^{-1 / 2} h_{K K}^{\mu \nu}\right)\left(R_{\mu \nu}^{(10)}-\frac{1}{2} g_{\mu \nu} R^{(10)}-\frac{1}{M_{(10)}^{8}} T_{\mu \nu}^{\text {matter }}\right)
$$

Under the metric (A.1),

$$
R_{\mu \nu}^{(10)}=R_{\mu \nu}^{(4)}-\frac{1}{2} \nabla_{(6)}^{2} g_{\mu \nu}-\frac{1}{4} g^{\rho \lambda} \partial_{m} g_{\mu \nu} \partial^{m} g_{\rho \lambda}+\frac{1}{2} g^{\rho \lambda} \partial_{m} g_{\nu \rho} \partial^{m} g_{\mu \lambda}
$$

where $R_{\mu \nu}^{(4)}$ is constructed from 4-d metric $g_{\mu \nu}$, and $\nabla_{(6)}$ is constructed from the internal metric $g_{m n}$. For the background warped geometry, the source does not depend on the large dimensions,

$$
\frac{\partial \mathcal{L}^{\text {matter }}}{\partial g^{\mu \nu}}=0
$$

Therefore, $T_{\mu \nu}^{\text {matter }}$ is proportional to $g_{\mu \nu}$,

$$
T_{\mu \nu}^{\text {matter }}=-\frac{1}{2} g_{\mu \nu} \mathcal{L}^{\text {matter }}
$$

Except for the one KK mode, all the other $\hat{g}_{\mu \nu}$ in (A.3) are gravitons and therefore $y$-independent. Hence in the last three terms of (A.4), $\hat{g}_{\mu \nu}$ factors out, and we get

$$
\begin{aligned}
& R_{\mu \nu}^{(10)}-\frac{1}{2} g_{\mu \nu} R^{(10)}-\frac{1}{M_{(10)}^{8}} T_{\mu \nu}^{\text {matter }} \\
= & \hat{R}_{\mu \nu}^{(4)}-\frac{1}{2} \hat{g}_{\mu \nu} \hat{R}^{(4)} \\
- & \hat{g}_{\mu \nu}\left(-\frac{1}{2} \nabla_{(6)}^{2} A^{-1 / 2}-\frac{1}{2} A^{1 / 2} \partial_{m} A^{-1 / 2} \partial^{m} A^{-1 / 2}+\frac{1}{2} A^{-1 / 2} R_{(6)}\right) \\
+ & \hat{g}_{\mu \nu} \frac{1}{2 M_{(10)}^{8}} A^{-1 / 2} \mathcal{L}^{\text {matter }},
\end{aligned}
$$

where $\hat{R}_{\mu \nu}^{(4)}$ is constructed from $\hat{g}_{\mu \nu}$. 
The last two terms in (A.7) cancel because the background equations of motion are just these two terms with the overall factor $\hat{g}_{\mu \nu}$ replaced by $\eta_{\mu \nu}$, the rest does not depend on $\hat{g}_{\mu \nu}$. The first two terms also cancel because the graviton wave-function satisfies $\hat{R}_{\mu \nu}^{(4)}-\frac{1}{2} \hat{g}_{\mu \nu} \hat{R}^{(4)}=0$ by definition, which is beyond the linear approximation and includes all possible non-linear couplings. Therefore (A.3) vanishes identically, namely, the decay product of one KK mode cannot be all gravitons. 


\section{References}

[1] G. R. Dvali and S.-H. H. Tye, "Brane inflation," Phys. Lett. B 450, 72 (1999) [arXiv:hep-ph/9812483].

[2] C. P. Burgess, M. Majumdar, D. Nolte, F. Quevedo, G. Rajesh and R. J. Zhang, "The inflationary brane-antibrane universe," JHEP 0107, 047 (2001) [arXiv:hep-th/0105204].

[3] G. R. Dvali, Q. Shafi and S. Solganik, "D-brane inflation," arXiv:hep-th/0105203.

[4] S. B. Giddings, S. Kachru and J. Polchinski, "Hierarchies from fluxes in string compactifications," Phys. Rev. D 66, 106006 (2002) [arXiv:hep-th/0105097].

[5] S. Kachru, R. Kallosh, A. Linde and S. P. Trivedi, "De Sitter vacua in string theory," Phys. Rev. D 68, 046005 (2003) [arXiv:hep-th/0301240].

[6] M. Grana, "Flux compactifications in string theory: A comprehensive review," Phys. Rept. 423, 91 (2006) [arXiv:hep-th/0509003].

[7] S. Kachru, R. Kallosh, A. Linde, J. Maldacena, L. McAllister and S. P. Trivedi, "Towards inflation in string theory," JCAP 0310, 013 (2003) [arXiv:hep-th/0308055].

[8] H. Firouzjahi and S.-H. H. Tye, "Brane inflation and cosmic string tension in superstring theory," JCAP 0503, 009 (2005) [arXiv:hep-th/0501099].

[9] E. Silverstein and D. Tong, "Scalar speed limits and cosmology: Acceleration from D-cceleration," Phys. Rev. D 70, 103505 (2004) [arXiv:hep-th/0310221].

[10] M. Alishahiha, E. Silverstein and D. Tong, "DBI in the sky," Phys. Rev. D 70, 123505 (2004) [arXiv:hep-th/0404084].

[11] X. G. Chen, "Multi-throat brane inflation," Phys. Rev. D 71, 063506 (2005) [arXiv:hep-th/0408084].

[12] X. G. Chen, "Inflation from warped space," JHEP 0508, 045 (2005) [arXiv:hep-th/0501184].

[13] S. E. Shandera and S.-H. H. Tye, "Observing brane inflation," arXiv:hep-th/0601099.

[14] S. Kachru, J. Pearson and H. Verlinde, "Brane/flux annihilation and the string dual of a non-supersymmetric field theory," JHEP 0206, 021 (2002) [arXiv:hep-th/0112197].

[15] O. DeWolfe, S. Kachru and H. Verlinde, "The giant inflaton," JHEP 0405, 017 (2004) [arXiv:hep-th/0403123].

[16] J. M. Cline and H. Stoica, "Multibrane inflation and dynamical flattening of the inflaton potential," Phys. Rev. D 72, 126004 (2005) [arXiv:hep-th/0508029].

[17] L. Randall and R. Sundrum, "A large mass hierarchy from a small extra dimension," Phys. Rev. Lett. 83, 3370 (1999) [arXiv:hep-ph/9905221]. 
[18] X. G. Chen, "Running non-Gaussianities in DBI inflation," Phys. Rev. D 72, 123518 (2005) [arXiv:astro-ph/0507053].

[19] N. Jones, H. Stoica and S.-H. H. Tye, "Brane interaction as the origin of inflation," JHEP 0207, 051 (2002) [arXiv:hep-th/0203163].

[20] S. Sarangi and S.-H. H. Tye, "Cosmic string production towards the end of brane inflation," Phys. Lett. B 536, 185 (2002) [arXiv:hep-th/0204074].

[21] E. J. Copeland, R. C. Myers and J. Polchinski, "Cosmic F- and D-strings," JHEP 0406, 013 (2004) [arXiv:hep-th/0312067].

[22] J. Polchinski, "Introduction to cosmic F- and D-strings," arXiv:hep-th/0412244.

[23] A. Sen, "Rolling tachyon," JHEP 0204, 048 (2002) [arXiv:hep-th/0203211].

[24] A. Sen, "Tachyon matter," JHEP 0207, 065 (2002) [arXiv:hep-th/0203265].

[25] A. Sen, "Tachyon dynamics in open string theory," Int. J. Mod. Phys. A 20, 5513 (2005) [arXiv:hep-th/0410103].

[26] G. Shiu, S.-H. H. Tye and I. Wasserman, "Rolling tachyon in brane world cosmology from superstring field theory," Phys. Rev. D 67, 083517 (2003) [arXiv:hep-th/0207119].

[27] J. M. Cline, H. Firouzjahi and P. Martineau, "Reheating from tachyon condensation," JHEP 0211, 041 (2002) [arXiv:hep-th/0207156].

[28] N. Lambert, H. Liu and J. Maldacena, "Closed strings from decaying D-branes," arXiv:hep-th/0303139.

[29] X. G. Chen, "One-loop evolution in rolling tachyon," Phys. Rev. D 70, 086001 (2004) [arXiv:hep-th/0311179].

[30] L. Leblond, "On the production of open strings from brane anti-brane annihilation," arXiv:hep-th/0510261.

[31] L. Leblond and S.-H. H. Tye, "Stability of D1-strings inside a D3-brane," JHEP 0403, 055 (2004) [arXiv:hep-th/0402072]

[32] J. Polchinski, "Open heterotic strings," arXiv:hep-th/0510033.

[33] J. J. Blanco-Pillado, G. Dvali and M. Redi, "Cosmic D-strings as axionic D-term strings," Phys. Rev. D 72, 105002 (2005) [arXiv:hep-th/0505172].

[34] N. Barnaby, C. P. Burgess and J. M. Cline, "Warped reheating in brane-antibrane inflation," JCAP 0504, 007 (2005) [arXiv:hep-th/0412040].

[35] L. Kofman and P. Yi, "Reheating the universe after string theory inflation," Phys. Rev. D 72, 106001 (2005) [arXiv:hep-th/0507257]. 
[36] D. Chialva, G. Shiu and B. Underwood, "Warped reheating in multi-throat brane inflation," arXiv:hep-th/0508229.

[37] A. R. Frey, A. Mazumdar and R. Myers, "Stringy effects during inflation and reheating," arXiv:hep-th/0508139.

[38] I. R. Klebanov and M. J. Strassler, "Supergravity and a confining gauge theory: Duality cascades and chiSB-resolution of naked singularities," JHEP 0008, 052 (2000) [arXiv:hep-th/0007191].

[39] M. Krasnitz, "A two point function in a cascading $\mathrm{N}=1$ gauge theory from supergravity," arXiv:hep-th/0011179.

[40] H. Firouzjahi and S.-H. H. Tye, "The shape of gravity in a warped deformed conifold," arXiv:hep-th/0512076.

[41] S. Dimopoulos, S. Kachru, N. Kaloper, A. E. Lawrence and E. Silverstein, "Small numbers from tunneling between brane throats," Phys. Rev. D 64, 121702 (2001) [arXiv:hep-th/0104239].

[42] S. Dimopoulos, S. Kachru, N. Kaloper, A. E. Lawrence and E. Silverstein, "Generating small numbers by tunneling in multi-throat compactifications," Int. J. Mod. Phys. A 19, 2657 (2004) [arXiv:hep-th/0106128].

[43] K. R. Dienes, E. Dudas and T. Gherghetta, "Grand unification at intermediate mass scales through extra dimensions," Nucl. Phys. B 537, 47 (1999) [arXiv:hep-ph/9806292].

[44] H. C. Cheng, J. L. Feng and K. T. Matchev, "Kaluza-Klein dark matter," Phys. Rev. Lett. 89, 211301 (2002) [arXiv:hep-ph/0207125].

[45] G. Servant and T. M. P. Tait, "Is the lightest Kaluza-Klein particle a viable dark matter candidate?," Nucl. Phys. B 650, 391 (2003) [arXiv:hep-ph/0206071].

[46] H. L. Verlinde, "Holography and compactification," Nucl. Phys. B 580, 264 (2000) [arXiv:hep-th/9906182].

[47] A. A. Starobinsky and S. M. Churilov, Sov. Phys. JETP 38, 1 (1974).

[48] G. W. Gibbons, "Vacuum Polarization And The Spontaneous Loss Of Charge By Black Holes," Commun. Math. Phys. 44, 245 (1975).

[49] D. N. Page, "Particle Emission Rates From A Black Hole: Massless Particles From An Phys. Rev. D 13, 198 (1976).

[50] W. G. Unruh, "Absorption Cross-Section Of Small Black Holes," Phys. Rev. D 14, 3251 (1976).

[51] A. Dhar, G. Mandal and S. R. Wadia, "Absorption vs decay of black holes in string theory and T-symmetry," Phys. Lett. B 388, 51 (1996) [arXiv:hep-th/9605234]. 
[52] S. R. Das, G. W. Gibbons and S. D. Mathur, "Universality of low energy absorption cross sections for black holes," Phys. Rev. Lett. 78, 417 (1997) [arXiv:hep-th/9609052].

[53] I. R. Klebanov, "World-volume approach to absorption by non-dilatonic branes," Nucl. Phys. B 496, 231 (1997) [arXiv:hep-th/9702076].

[54] E. Merzbacher, Quantum Mechanics, John Wiley \& Sons, 1970.

[55] R. Iengo and J. G. Russo, "Handbook on string decay," arXiv:hep-th/0601072.

[56] J. Garcia-Bellido, R. Rabadan and F. Zamora, "Inflationary scenarios from branes at angles," JHEP 0201, 036 (2002) [arXiv:hep-th/0112147].

[57] K. Dasgupta, C. Herdeiro, S. Hirano and R. Kallosh, "D3/D7 inflationary model and M-theory," Phys. Rev. D 65, 126002 (2002) [arXiv:hep-th/0203019].

[58] A. Buchel, "Gauge / gravity correspondence in accelerating universe," Phys. Rev. D 65, 125015 (2002) [arXiv:hep-th/0203041].

[59] S. B. Giddings and A. Maharana, "Dynamics of warped compactifications and the shape of the warped landscape," arXiv:hep-th/0507158.

[60] R. D. Peccei and H. R. Quinn, "Constraints Imposed By CP Conservation In The Presence Of Instantons," Phys. Rev. D 16, 1791 (1977).

[61] F. Wilczek, "Problem Of Strong P And T Invariance In The Presence Of Instantons," Phys. Rev. Lett. 40, 279 (1978).

[62] S. Weinberg, "A New Light Boson?," Phys. Rev. Lett. 40, 223 (1978).

[63] H. Goldberg, "Constraint On The Photino Mass From Cosmology," Phys. Rev. Lett. 50, 1419 (1983).

[64] S. H. Tye, I. Wasserman and M. Wyman, "Scaling of multi-tension cosmic superstring networks," Phys. Rev. D 71, 103508 (2005) [Erratum-ibid. D 71, 129906 (2005)] [arXiv:astro-ph/0503506].

[65] J. R. Ellis, J. S. Hagelin, D. V. Nanopoulos, K. A. Olive and M. Srednicki, "Supersymmetric Relics From The big bang," Nucl. Phys. B 238, 453 (1984).

[66] K. Griest and M. Kamionkowski, "Unitarity Limits On The Mass And Radius Of Dark Matter Particles," Phys. Rev. Lett. 64, 615 (1990).

[67] K. Greisen, "End To The Cosmic Ray Spectrum?," Phys. Rev. Lett. 16, 748 (1966).

[68] G. T. Zatsepin and V. A. Kuzmin, "Upper Limit Of The Spectrum Of Cosmic Rays," JETP Lett. 4, 78 (1966) [Pisma Zh. Eksp. Teor. Fiz. 4, 114 (1966)].

[69] J. L. Feng, A. Rajaraman and F. Takayama, "Superweakly-interacting massive particles," Phys. Rev. Lett. 91, 011302 (2003) [arXiv:hep-ph/0302215]. 
[70] D. J. H. Chung, E. W. Kolb and A. Riotto, "Nonthermal supermassive dark matter," Phys. Rev. Lett. 81, 4048 (1998) [arXiv:hep-ph/9805473].

[71] D. M. Wittman, J. A. Tyson, D. Kirkman, I. Dell'Antonio and G. Bernstein, "Detection of weak gravitational lensing distortions of distant galaxies by cosmic dark matter at large scales," Nature 405, 143 (2000) [arXiv:astro-ph/0003014].

[72] L. McAllister and I. Mitra, "Relativistic D-brane scattering is extremely inelastic," JHEP 0502, 019 (2005) [arXiv:hep-th/0408085]. 